\title{
HIGHER ORDER ENERGY CONSERVATION AND GLOBAL WELLPOSEDNESS OF SOLUTIONS FOR GROSS-PITAEVSKII HIERARCHIES
}

\author{
THOMAS CHEN AND NATAŠA PAVLOVIĆ
}

\begin{abstract}
We consider the cubic and quintic Gross-Pitaevskii (GP) hierarchies in $d$ dimensions, for focusing and defocusing interactions. We introduce new higher order energy functionals and prove that they are conserved for solutions of energy subcritical defocusing, and $L^{2}$ subcritical (de)focusing GP hierarchies, in spaces also used by Erdös, Schlein and Yau in 11 12. By use of this tool, we prove a priori $H^{1}$ bounds for positive semidefinite solutions in those spaces. Moreover, we obtain global well-posedness results for positive semidefinite solutions in the spaces studied in the works of Klainerman and Machedon, 20], and in [8]. As part of our analysis, we prove generalizations of Sobolev and Gagliardo-Nirenberg inequalities for density matrices.
\end{abstract}

\section{INTRODUCTION}

In recent years, there has been impressive progress related to the derivation of nonlinear dispersive PDEs, such as the nonlinear Schrödinger (NLS) or nonlinear Hartree (NLH) equations, from many body quantum dynamics, see [11, 12, 13, 21, 20, 25] and the references therein, and also [1, 3, 10, 14, 15, 16, 18, 17, 19, 27. Closely related to this research field is the mathematical study of Bose-Einstein condensation in systems of interacting bosons, where we refer to the highly influencial works [2, 22, 23, 24] and the references therein.

1.1. The Gross-Pitaevkii limit for Bose gases. In the landmark works [1, 12, 13], Erdös, Schlein, and Yau developed a powerful method to derive the NLS as a dynamical Gross-Pitaevskii limit of an interacting Bose gas. For the convenience of the reader, and as a preparation for our discussion below, we will here outline some of the main steps, following [11, 12, 13. For the derivation of the defocusing quintic NLS from a system of bosons with repelling three body interactions, we refer to 7 .

1.1.1. From $N$-body Schrödinger to BBGKY hierarchy. We consider a system of $N$ bosons in $\mathbb{R}^{d}$ where $\psi_{N} \in L^{2}\left(\mathbb{R}^{d N}\right)$ denotes its wave function. To comply with Bose-Einstein statistics, $\psi_{N}$ is invariant under the permutation of particle variables,

$$
\psi_{N}\left(x_{\pi(1)}, x_{\pi(2)}, \ldots, x_{\pi(N)}\right)=\psi_{N}\left(x_{1}, x_{2}, \ldots, x_{N}\right) \quad \forall \pi \in S_{N},
$$

where $S_{N}$ is the $N$-th symmetric group. We denote by $L_{s}^{2}\left(\mathbb{R}^{d N}\right)$ the subspace of $L^{2}\left(\mathbb{R}^{d N}\right)$ of elements obeying (1.1). The dynamics of the system is determined by 
the $N$-body Schrödinger equation

$$
i \partial_{t} \psi_{N}=H_{N} \psi_{N}
$$

The Hamiltonian $H_{N}$ is assumed to be a self-adjoint operator acting on the Hilbert space $L_{s}^{2}\left(\mathbb{R}^{d N}\right)$, given by

$$
H_{N}=\sum_{j=1}^{N}\left(-\Delta_{x_{j}}\right)+\frac{1}{N} \sum_{1 \leq i<j \leq N} V_{N}\left(x_{i}-x_{j}\right),
$$

where $V_{N}(x)=N^{d \beta} V\left(N^{\beta} x\right)$ with $V \in W^{r, s}\left(\mathbb{R}^{d}\right)$ spherically symmetric, for some suitable $r, s$, and for $\beta \in(0,1]$ sufficiently small].

Since the Schrödinger equation (1.2) is linear and $H_{N}$ self-adjoint, the global wellposedness of solutions is satisfied. However, due to the exceedingly large number of degrees of freedom of order $O(N)$ (varying from $N \sim 10^{3}$ for samples of very dilute Bose-Einstein gases, to $N \sim 10^{30}$ in boson stars), it is very difficult to understand qualitative and quantitative properties of the bulk dynamics of the system, if directly based on the solution of (1.2). It is often much more informative to study coarse grained, effective properties of the system obtained from averaging over large $N$. In addition, taking the limit $N \rightarrow \infty$ for an appropriate scaling typically leads to macroscopic effective theories, or mean-field theories which are expected to accurately describe properties of the underlying physical system with very large, but finite $N$.

To perform the infinite particle number limit $N \rightarrow \infty$, we consider the strategy developed in 11, 12, which can be described as follows. First of all, one introduces the density matrix

$$
\gamma_{N}\left(t, \underline{x}_{N}, \underline{x}_{N}^{\prime}\right)=\psi_{N}\left(t, \underline{x}_{N}\right) \overline{\psi_{N}\left(t, \underline{x}_{N}^{\prime}\right)}
$$

where $\underline{x}_{N}=\left(x_{1}, x_{2}, \ldots, x_{N}\right)$ and $\underline{x}_{N}^{\prime}=\left(x_{1}^{\prime}, x_{2}^{\prime}, \ldots, x_{N}^{\prime}\right)$. Furthermore, one considers the associated sequence of $k$-particle marginal density matrice $3 \gamma_{N}^{(k)}(t)$, for $k=$ $1, \ldots, N$, as the partial traces of $\gamma_{N}$ over the degrees of freedom associated to the last $(N-k)$ particle variables,

$$
\gamma_{N}^{(k)}=\operatorname{Tr}_{k+1, k+2, \ldots, N}\left|\psi_{N}\right\rangle\left\langle\psi_{N}\right| .
$$

Here, $\operatorname{Tr}_{k+1, k+2, \ldots, N}$ denotes the partial trace with respect to the particles indexed by $k+1, k+2, \ldots, N$. Accordingly, $\gamma_{N}^{(k)}$ is explicitly given by

$$
\begin{aligned}
\gamma_{N}^{(k)}\left(\underline{x}_{k}, \underline{x}_{k}^{\prime}\right) & =\int d \underline{x}_{N-k} \gamma_{N}\left(\underline{x}_{k}, \underline{x}_{N-k} ; \underline{x}_{k}^{\prime}, \underline{x}_{N-k}\right) \\
& =\int d \underline{x}_{N-k} \overline{\psi_{N}\left(\underline{x}_{k}, \underline{x}_{N-k}\right)} \psi_{N}\left(\underline{x}_{k}^{\prime}, \underline{x}_{N-k}\right) .
\end{aligned}
$$

\footnotetext{
${ }^{1}$ When $\beta=1$, the Hamiltonian 1.3 is called the Gross-Pitaevskii Hamiltonian. Due to the factor $\frac{1}{N}$ in front of the interaction potential, 1.3) can be formally interpreted as a mean field Hamiltonian. However, it should be noted that (1.3) in fact describes a very dilute gas, where interactions among particles are very rare and strong, while in a mean field Hamiltonian each particle usually reacts with all other particles via a very weak potential. However, one can still apply to (1.3) similar mathematical methods as in the case of a mean field potential.

${ }^{2}$ The $k$-particle marginal density matrices play a key role in the analysis of the system as $N \rightarrow \infty$, because for every fixed $k, \gamma_{N}^{(k)}$ can have a well defined limit.
} 
It is clear from the definitions that the property of admissibility holds,

$$
\gamma_{N}^{(k)}=\operatorname{Tr}_{k+1} \gamma_{N}^{(k+1)} \quad, \quad k=1, \ldots, N-1,
$$

and that $\operatorname{Tr} \gamma_{N}^{(k)}=\left\|\psi_{N}\right\|_{L_{s}^{2}\left(\mathbb{R}^{d N}\right)}^{2}=1$ for all $N$, and all $k=1,2, \ldots, N$.

Moreover, $\gamma_{N}^{(k)} \geq 0$ is positive semidefinite as an operator $\mathcal{S}\left(\mathbb{R}^{k d}\right) \times \mathcal{S}\left(\mathbb{R}^{k d}\right) \rightarrow \mathbb{C}$, $(f, g) \mapsto \int d \underline{x} d \underline{x}^{\prime} f(\underline{x}) \gamma\left(\underline{x} ; \underline{x}^{\prime}\right) \overline{g\left(\underline{x}^{\prime}\right)}$.

The time evolution of the density matrix $\gamma_{N}$ is determined by the Heisenberg equation

$$
i \partial_{t} \gamma_{N}(t)=\left[H_{N}, \gamma_{N}(t)\right]
$$

which has the explicit form

$$
\begin{aligned}
i \partial_{t} \gamma_{N}\left(t, \underline{x}_{N}, \underline{x}_{N}^{\prime}\right)= & -\left(\Delta_{\underline{x}_{N}}-\Delta_{\underline{x}_{N}^{\prime}}\right) \gamma_{N}\left(t, \underline{x}_{N}, \underline{x}_{N}^{\prime}\right) \\
& +\frac{1}{N} \sum_{1 \leq i<j \leq N}\left[V_{N}\left(x_{i}-x_{j}\right)-V_{N}\left(x_{i}^{\prime}-x_{j}^{\prime}\right)\right] \gamma_{N}\left(t, \underline{x}_{N}, \underline{x}_{N}^{\prime}\right)
\end{aligned}
$$

Accordingly, the $k$-particle marginals satisfy the BBGKY hierarchy

$$
\begin{aligned}
& i \partial_{t} \gamma^{(k)}\left(t, \underline{x}_{k} ; \underline{x}_{k}^{\prime}\right)=-\left(\Delta_{\underline{x}_{k}}-\Delta_{\underline{x}_{k}^{\prime}}\right) \gamma^{(k)}\left(t, \underline{x}_{k}, \underline{x}_{k}^{\prime}\right) \\
& +\frac{1}{N} \sum_{1 \leq i<j \leq k}\left[V_{N}\left(x_{i}-x_{j}\right)-V_{N}\left(x_{i}^{\prime}-x_{j}^{\prime}\right)\right] \gamma^{(k)}\left(t, \underline{x}_{k} ; \underline{x}_{k}^{\prime}\right) \\
& +\frac{N-k}{N} \sum_{i=1}^{k} \int d x_{k+1}\left[V_{N}\left(x_{i}-x_{k+1}\right)-V_{N}\left(x_{i}^{\prime}-x_{k+1}\right)\right] \\
& \gamma^{(k+1)}\left(t, \underline{x}_{k}, x_{k+1} ; \underline{x}_{k}, x_{k+1}^{\prime}\right)
\end{aligned}
$$

where $\Delta_{\underline{x}_{k}}:=\sum_{j=1}^{k} \Delta_{x_{j}}$, and similarly for $\Delta_{\underline{x}_{k}^{\prime}}$. We note that the number of terms in (1.8) is $\approx \frac{k^{2}}{N} \rightarrow 0$, and the number of terms in (1.9) is $\frac{k(N-k)}{N} \rightarrow k$ as $N \rightarrow \infty$. Accordingly, for fixed $k$, (1.8) disappears in the limit $N \rightarrow \infty$ described below, while (1.9) survives.

1.1.2. From BBGKY hierarchy to GP hierarchy. A crucial step in this analysis is the limit $N \rightarrow \infty$, and the extraction of closed equations for the time evolution of $\lim _{N \rightarrow \infty} \gamma_{N}^{(k)}$. It is proven in [11, 12, 13] that, for asymptotically factorizing initial data, and a suitable weak topology on the space of marginal density matrices, one can extract convergent subsequences $\gamma_{N}^{(k)} \rightarrow \gamma^{(k)}$ as $N \rightarrow \infty$, for $k \in \mathbb{N}$, which satisfy the the infinite limiting hierarchy

$$
\begin{aligned}
i \partial_{t} \gamma^{(k)}\left(t, \underline{x}_{k} ; \underline{x}_{k}^{\prime}\right)= & -\left(\Delta_{\underline{x}_{k}}-\Delta_{\underline{x}_{k}^{\prime}}\right) \gamma^{(k)}\left(t, \underline{x}_{k} ; \underline{x}_{k}^{\prime}\right) \\
& +b_{0} \sum_{j=1}^{k}\left(B_{j, k+1} \gamma^{k+1}\right)\left(t, \underline{x}_{k} ; \underline{x}_{k}^{\prime}\right),
\end{aligned}
$$

which is referred to as the Gross-Pitaevskii (GP) hierarchy. Here,

$$
\begin{aligned}
& \left(B_{j, k+1} \gamma^{k+1}\right)\left(t, \underline{x}_{k} ; \underline{x}_{k}^{\prime}\right) \\
& :=\int d x_{k+1} d x_{k+1}^{\prime}\left[\delta\left(x_{j}-x_{k+1}\right) \delta\left(x_{j}-x_{k+1}^{\prime}\right)-\delta\left(x_{j}^{\prime}-x_{k+1}\right) \delta\left(x_{j}^{\prime}-x_{k+1}^{\prime}\right)\right] \\
& \gamma^{(k+1)}\left(t, \underline{x}_{k}, x_{k+1} ; \underline{x}_{k}^{\prime}, x_{k+1}^{\prime}\right) .
\end{aligned}
$$


The coefficient $b_{0}$ is the scattering length if $\beta=1$ (see [11, 23. for the definition), and $b_{0}=\int V(x) d x$ if $\beta<1$ (corresponding to the Born approximation of the scattering length). For $\beta<1$, the interaction term is obtained from the weak limit $V_{N}(x) \rightarrow b_{0} \delta(x)$ in (1.9) as $N \rightarrow \infty$. The proof for the case $\beta=1$ is much more difficult, and the derivation of the scattering length in this context is a breakthrough result obtained in [11, 12. For notational convenience, we will mostly set $b_{0}=1$ in the sequel.

Some key properties satisfied by the solutions of the GP hierarchy are:

- The solution of the GP hierarchy obtained in [11, 12, exists globally in $t$.

- It satisfies the property of admissibility,

$$
\gamma^{(k)}=\operatorname{Tr}_{k+1} \gamma^{(k+1)} \quad, \quad \forall k \in \mathbb{N}
$$

which is inherited from the system at finite $N$.

- There exists a constant $C_{0}$ depending on the initial data only, such that the a priori energy bound

$$
\operatorname{Tr}\left(\left|S^{(k, 1)} \gamma^{(k)}(t)\right|\right)<C_{0}^{k}
$$

is satisfied for all $k \in \mathbb{N}$, and for all $t \in \mathbb{R}$, where

$$
S^{(k, \alpha)}:=\prod_{j=1}^{k}\left\langle\nabla_{x_{j}}\right\rangle^{\alpha}\left\langle\nabla_{x_{j}^{\prime}}\right\rangle^{\alpha} .
$$

This is obtained from energy conservation in the original $N$-body Schrödinger system.

- Solutions of the GP hierarchy are studied in spaces of $k$-particle marginals $\left\{\gamma^{(k)} \mid\left\|\gamma^{(k)}\right\|_{\mathfrak{h}^{1}}<\infty\right\}$ with norms

$$
\left\|\gamma^{(k)}\right\|_{\mathfrak{h}^{\alpha}}:=\operatorname{Tr}\left(\left|S^{(k, \alpha)} \gamma^{(k)}\right|\right) \text {. }
$$

This is in agreement with the a priori bounds (1.12).

1.1.3. Factorized solutions of GP and NLS. The NLS emerges as the mean field dynamics of the Bose gas for the very special subclass of solutions of the GP hierarchy that are factorized. Factorized $k$-particle marginals at time $t=0$ have the form

$$
\left.\gamma_{0}^{(k)}\left(\underline{x}_{k} ; \underline{x}_{k}^{\prime}\right)=\prod_{j=1}^{k} \phi_{0}\left(x_{j}\right) \overline{\phi_{0}\left(x_{j}^{\prime}\right.}\right),
$$

where we assume that $\phi_{0} \in H^{1}\left(\mathbb{R}^{d}\right)$. One can easily verify that the solution of the GP hierarchy remains factorized for all $t \in I \subseteq \mathbb{R}$,

$$
\gamma^{(k)}\left(t, \underline{x}_{k} ; \underline{x}_{k}^{\prime}\right)=\prod_{j=1}^{k} \phi\left(t, x_{j}\right) \overline{\phi\left(t, x_{j}^{\prime}\right)},
$$

and that for the GP hierarchy given in (1.10) with $b_{0}=1, \phi(t) \in H^{1}\left(\mathbb{R}^{d}\right)$ solves the defocusing cubic NLS,

$$
i \partial_{t} \phi=-\Delta_{x} \phi+|\phi|^{2} \phi,
$$

for $t \in I \subseteq \mathbb{R}$, and $\phi(0)=\phi_{0} \in H^{1}\left(\mathbb{R}^{d}\right)$. 
1.1.4. Uniqueness of solutions of GP hierarchies. While the existence of factorized solutions can be easily verified in the manner outlined above, the proof of the uniqueness of solutions of the GP hierarchy (which encompass non-factorized solutions) is the most difficult part in this analysis. The proof of uniqueness of solutions to the GP hierarchy was originally achieved by Erdös, Schlein and Yau in 11, 12, 13, in the space $\left\{\gamma^{(k)} \mid\left\|\gamma^{(k)}\right\|_{\mathfrak{h}^{1}}<\infty\right\}$, for which the authors developed highly sophisticated Feynman graph expansion methods.

In 20, Klainerman and Machedon introduced an alternative method for proving uniqueness in a space of density matrices defined by the Hilbert-Schmidt type Sobolev norms

$$
\left\|\gamma^{(k)}\right\|_{H_{k}^{\alpha}}:=\left(\operatorname{Tr}\left(\left|S^{(k, \alpha)} \gamma^{(k)}\right|^{2}\right)\right)^{\frac{1}{2}}<\infty .
$$

While this is a different (strictly larger) space of marginal density matrices than the one considered by Erdös, Schlein, and Yau, 11, 12, the authors of 20] impose an additional a priori condition on space-time norms of the form

$$
\left\|B_{j ; k+1} \gamma^{(k+1)}\right\|_{L_{t}^{2} H_{k}^{1}}<C^{k},
$$

for some arbitrary but finite $C$ independent of $k$. The strategy in 20] developed to prove the uniqueness of solutions of the GP hierarchy (1.10) in $d=3$ involves the use of certain space-time bounds on density matrices (of generalized Strichartz type), and crucially employs the reformulation of a combinatorial result in [11, 12] into a "board game" argument. The latter is used to organize the Duhamel expansion of solutions of the GP hierarchy into equivalence classes of terms which leads to a significant reduction of the complexity of the problem.

Subsequently, Kirkpatrick, Schlein, and Staffilani proved in 21] that the a priori spacetime bound (1.17) is satisfied for the cubic GP hierarchy in $d=2$, locally in time. Their argument is based on the conservation of energy in the original $N$-body Schrödinger system, and a related a priori $H^{1}$-bounds for the BBGKY hierarchy in the limit $N \rightarrow \infty$ derived in [11, 12, combined with a generalized Sobolev inequality for density matrices.

1.2. On the Cauchy problem for GP hierarchies. It is currently not known how to rigorously derive a GP hierarchy from the $N \rightarrow \infty$ limit of a BBGKY hierarchy with $L^{2}$-supercritical, attractive interactions. Nevertheless, we have begun in 8 . to adopt as our starting point the level of GP hierarchies, and to study the well-posedness of the Cauchy problem for systems with both focusing and defocusing interactions. Accordingly, the corresponding GP hierarchies are referred to as cubic, quintic, focusing, or defocusing GP hierarchies, depending on the type of the NLS governing the solutions obtained from factorized initial conditions.

In [8, we introduced the following topology on the Banach space of sequences of $k$-particle marginal density matrices

$$
\mathfrak{G}=\left\{\Gamma=\left(\gamma^{(k)}\left(x_{1}, \ldots, x_{k} ; x_{1}^{\prime}, \ldots, x_{k}^{\prime}\right)\right)_{k \in \mathbb{N}} \mid \operatorname{Tr} \gamma^{(k)}<\infty\right\} .
$$

Given $\xi>0$, we defined the space

$$
\mathcal{H}_{\xi}^{\alpha}=\left\{\Gamma \mid\|\Gamma\|_{\mathcal{H}_{\xi}^{\alpha}}<\infty\right\}
$$


with the norm

$$
\|\Gamma\|_{\mathcal{H}_{\xi}^{\alpha}}:=\sum_{k \in \mathbb{N}} \xi^{k}\left\|\gamma^{(k)}\right\|_{H^{\alpha}}
$$

where

$$
\left\|\gamma^{(k)}\right\|_{H_{k}^{\alpha}}:=\operatorname{Tr}\left(\left|S^{(k, \alpha)} \gamma^{(k)}\right|^{2}\right)^{\frac{1}{2}}
$$

is the norm (1.16) considered in 20. If $\Gamma \in \mathcal{H}_{\xi}^{\alpha}$, then $\xi^{-1}$ an upper bound on the typical $H^{\alpha}$-energy per particle; this notion is made precise in $[8$. We note that small energy results are characterized by large $\xi>1$, while results valid without any upper bound on the size of the energy can be proven for arbitrarily small values of $\xi>0$; in the latter case, one can assume $0<\xi<1$ without any loss of generality. The parameter $\alpha$ determines the regularity of the solution.

In [8], we prove the local well-posedness of solutions for energy subcritical focusing and defocusing cubic and quintic GP hierarchies in a subspace of $\mathcal{H}_{\xi}^{\alpha}$ defined by a condition related to (1.17) 3 . The precise formulation is given in Theorem 3.4 below. Our result is obtained from a Picard fixed point argument, and holds for various dimensions $d$, without any requirement on factorization. The parameter $\xi>0$ is determined by the initial condition, and it sets the energy scale of the given Cauchy problem. In addition, we prove lower bounds on the blowup rate for blowup solutions of focusing GP-hierarchies in [8].

In the joint work [9] with Tzirakis, we identify a conserved energy functional $E_{1}(\Gamma(t))=E_{1}\left(\Gamma_{0}\right)$ describing the average energy per particle (the precise definition is given in (4.2) below), and we prove virial identities for solutions of GP hierarchies. In particular, we use these ingredients to prove that for $L^{2}$-critical and supercritical focusing GP hierarchies, blowup occurs whenever $E_{1}\left(\Gamma_{0}\right)<0$ and the variance is finite. We note that prior to [9], no exact conserved energy functional on the level of the GP hierarchy was identified in any of the previous works, including [21] and [11, 12.

1.3. Main results of this paper. We emphasize again that our results in $[8$, which are quoted in Theorem 3.4 below, imply the local well-posedness of solutions for the spaces considered by Klainerman and Machedon, [20; those are of the form $\mathcal{H}_{\xi}^{\alpha}$, under constraints similar to (1.17). However, they do not hold for the spaces considered by Erdös, Schlein and Yau, [11, 12, which are of the form $\mathfrak{H}_{\xi}^{1}$ introduced below.

To be more precise, we define the spaces

$$
\mathfrak{H}_{\xi}^{\alpha}=\left\{\Gamma \in \mathfrak{G} \mid\|\Gamma\|_{\mathfrak{H}_{\xi}^{\alpha}}<\infty\right\}
$$

with

$$
\|\Gamma\|_{\mathfrak{H}_{\xi}^{\alpha}}:=\sum_{k \in \mathbb{N}} \xi^{k}\left\|\gamma^{(k)}\right\|_{\mathfrak{h}^{\alpha}}
$$

where

$$
\left\|\gamma^{(k)}\right\|_{\mathfrak{h}^{\alpha}}:=\operatorname{Tr}\left(\left|S^{(k, \alpha)} \gamma^{(k)}\right|\right) .
$$

\footnotetext{
3 The parameter $\alpha$ determines the regularity of the solution (e.g. for cubic GP when $d=3$, $\alpha \in[1, \infty))$.
} 
For the existence of solutions $\Gamma(t) \in \mathfrak{H}_{\xi}^{1}$, we invoke the above noted results of Erdös, Schlein and Yau, [11, 12, where a global in time solution is constructed from the solution of an $N$-body Schrodinger equation and the associated BBGKY hierarchy. We note that expressed in our notation, the a priori energy bound (1.12) implies that for any $0<\xi<C_{0}^{-1}$, one has

$$
\|\Gamma(t)\|_{\mathfrak{H}_{\xi}^{1}}<\sum_{k \geq 1}\left(C_{0} \xi\right)^{k}<\infty \quad, \quad t \in \mathbb{R} .
$$

Hence, the solutions of the cubic defocusing GP hierarchy derived by Erdös, Schlein and Yau in [11, 12, are contained in the spaces $\mathfrak{H}_{\xi}^{1}$ considered in this paper. Similarly, solutions of the quintic GP hierarchy obtained in [7] (along the lines described in Section 1.1) are contained in $\mathfrak{H}_{\xi}^{1}$.

The main results proven in this paper are:

(1) We introduce a new family of higher order energy functionals, generalizing those found in 9, and prove that they are conserved for solutions of the GP hierarchy (see Section 4).

(2) We introduce a generalization of Sobolev and Gagliardo-Nirenberg inequalities on the level of marginal density matrices (see Section [5).

(3) We prove a priori energy bounds on positive semidefinite solutions $\Gamma(t) \in \mathfrak{H}_{\xi}^{1}$ for defocusing, energy-subcritical GP hierarchies (see Subsection 6.2), and for focusing, $L^{2}$-subcritical GP hierarchies (see Subsection 6.3). Our argument is based on the conservation of the higher order energy functionals, and employs the above noted Sobolev inequalities for marginal density matrices (see Section 5).

(4) Finally, we use the the higher order energy functionals in order to enhance local to global wellposedness for solutions in the spaces $\mathcal{H}_{\xi}^{1}$ constructed in 8], for initial data in $\mathfrak{H}_{\xi}^{1}$ (see Section 7).

We note that our a priori upper bounds on the norm $\|\Gamma\|_{\mathfrak{H}_{\xi}^{1}}$ hold for positive semidefinite $\Gamma$. The condition of positive definiteness is physically meaningful because the components of $\Gamma$ are interpreted as density matrices. An obvious example of such $\Gamma$ is given by arbitrary linear superpositions of factorized states with positive coefficients, $\Gamma_{0}=\sum \mu_{j} \Gamma_{\phi_{j}}, \mu_{j}>0$, and $\Gamma_{\phi_{j}}=\left(\left(\left|\phi_{j}\right\rangle\left\langle\phi_{j}\right|\right)^{\otimes n}\right)_{n \in \mathbb{N}}, \phi_{j} \in H^{1}\left(\mathbb{R}^{d}\right)$. Also, $\Gamma(t)$ obtained from the $N \rightarrow \infty$ limit of the BBGKY hierarchy of an $N$-body Schrödinger equation is generally expected to be positive semidefinite. However, the question whether positive semidefiniteness is generally preserved by the flow of the GP hierarchy or not is not well understood, as far as we know, and we leave a more systematic study of this issue to future work. 


\section{Definition OF THE MODEL}

In this section, we introduce the mathematical model analyzed in this paper. Most notations and definitions are adopted from [8], where we refer for motivations and more details.

2.1. The spaces of marginal density matrices. We consider the space introduced in 8

$$
\mathfrak{G}:=\bigoplus_{k=1}^{\infty} L^{2}\left(\mathbb{R}^{d k} \times \mathbb{R}^{d k}\right)
$$

of sequences of density matrices

$$
\Gamma:=\left(\gamma^{(k)}\right)_{k \in \mathbb{N}}
$$

where $\gamma^{(k)} \geq 0, \operatorname{Tr} \gamma^{(k)}=1$, and where every $\gamma^{(k)}\left(\underline{x}_{k}, \underline{x}_{k}^{\prime}\right)$ is symmetric in all components of $\underline{x}_{k}$, and in all components of $\underline{x}_{k}^{\prime}$, respectively, i.e.

$$
\gamma^{(k)}\left(x_{\pi(1)}, \ldots, x_{\pi(k)} ; x_{\pi^{\prime}(1)}^{\prime}, \ldots, x_{\pi^{\prime}(k)}^{\prime}\right)=\gamma^{(k)}\left(x_{1}, \ldots, x_{k} ; x_{1}^{\prime}, \ldots, x_{k}^{\prime}\right)
$$

holds for all $\pi, \pi^{\prime} \in S_{k}$.

Throughout this paper, we will denote vectors $\left(x_{1}, \cdots, x_{k}\right)$ by $\underline{x}_{k}$ and vectors $\left(x_{1}^{\prime}, \cdots, x_{k}^{\prime}\right)$ by $\underline{x}_{k}^{\prime}$.

The $k$-particle marginals are assumed to be hermitean,

$$
\gamma^{(k)}\left(\underline{x}_{k} ; \underline{x}_{k}^{\prime}\right)=\overline{\gamma^{(k)}\left(\underline{x}_{k}^{\prime} ; \underline{x}_{k}\right)}
$$

We call $\Gamma=\left(\gamma^{(k)}\right)_{k \in \mathbb{N}}$ admissible if $\gamma^{(k)}=\operatorname{Tr}_{k+1} \gamma^{(k+1)}$, that is,

$$
\gamma^{(k)}\left(\underline{x}_{k} ; \underline{x}_{k}^{\prime}\right)=\gamma^{(k+1)}\left(\underline{x}_{k}, x_{k+1} ; \underline{x}_{k}^{\prime}, x_{k+1}\right)
$$

for all $k \in \mathbb{N}$.

Let $0<\xi<1$. In $[8$, we introduced the Hilbert-Schmidt type generalized Sobolev spaces of sequences of marginal density matrices

$$
\mathcal{H}_{\xi}^{\alpha}:=\left\{\Gamma \in \mathfrak{G} \mid\|\Gamma\|_{\mathcal{H}_{\xi}^{\alpha}}<\infty\right\},
$$

where

$$
\|\Gamma\|_{\mathcal{H}_{\xi}^{\alpha}}=\sum_{k=1}^{\infty} \xi^{k}\left\|\gamma^{(k)}\right\|_{H_{k}^{\alpha}}
$$

with

$$
\left\|\gamma^{(k)}\right\|_{H_{k}^{\alpha}}:=\left(\operatorname{Tr}\left(\left|S^{(k, \alpha)} \gamma^{(k)}\right|^{2}\right)\right)^{\frac{1}{2}}
$$

and $S^{(k, \alpha)}:=\prod_{j=1}^{k}\left\langle\nabla_{x_{j}}\right\rangle^{\alpha}\left\langle\nabla_{x_{j}^{\prime}}\right\rangle^{\alpha}$. They also correspond to the spaces of solutions studied in [20].

In contrast, we also define the $\mathcal{L}^{1}$-Schatten class type generalized Sobolev spaces

$$
\mathfrak{H}_{\xi}^{\alpha}:=\left\{\Gamma \in \mathfrak{G} \mid\|\Gamma\|_{\mathfrak{H}_{\xi}^{\alpha}}<\infty\right\},
$$


where

$$
\|\Gamma\|_{\mathfrak{H}_{\xi}^{\alpha}}=\sum_{k=1}^{\infty} \xi^{k}\left\|\gamma^{(k)}\right\|_{\mathfrak{h}_{k}^{\alpha}}
$$

with

$$
\left\|\gamma^{(k)}\right\|_{\mathfrak{h}_{k}^{\alpha}}:=\operatorname{Tr}\left(\left|S^{(k, \alpha)} \gamma^{(k)}\right|\right)
$$

that correspond to the spaces of solutions studied in [11, 12].

2.2. The Gross-Pitaevskii (GP) hierarchy. To unify the notation for cubic and quintic GP hierarchies, we introduced the notion of $p$-GP hierarchy in [8], given as follows. Let $p \in\{2,4\}$. Then, the $p$-GP hierarchy is given by

$$
i \partial_{t} \gamma^{(k)}=\sum_{j=1}^{k}\left[-\Delta_{x_{j}}, \gamma^{(k)}\right]+\mu B_{k+\frac{p}{2}} \gamma^{\left(k+\frac{p}{2}\right)}
$$

for $k \in \mathbb{N}$. Here,

$$
B_{k+\frac{p}{2}} \gamma^{\left(k+\frac{p}{2}\right)}=B_{k+\frac{p}{2}}^{+} \gamma^{\left(k+\frac{p}{2}\right)}-B_{k+\frac{p}{2}}^{-} \gamma^{\left(k+\frac{p}{2}\right)},
$$

where

$$
B_{k+\frac{p}{2}}^{+} \gamma^{\left(k+\frac{p}{2}\right)}=\sum_{j=1}^{k} B_{j ; k+1, \ldots, k+\frac{p}{2}}^{+} \gamma^{\left(k+\frac{p}{2}\right)},
$$

and

$$
B_{k+\frac{p}{2}}^{-} \gamma^{\left(k+\frac{p}{2}\right)}=\sum_{j=1}^{k} B_{j ; k+1, \ldots, k+\frac{p}{2}}^{-} \gamma^{\left(k+\frac{p}{2}\right)},
$$

with

$$
\begin{aligned}
& \left(B_{j ; k+1, \ldots, k+\frac{p}{2}}^{+} \gamma^{\left(k+\frac{p}{2}\right)}\right)\left(t, x_{1}, \ldots, x_{k} ; x_{1}^{\prime}, \ldots, x_{k}^{\prime}\right) \\
& \quad=\int d x_{k+1} \cdots d x_{k+\frac{p}{2}} d x_{k+1}^{\prime} \cdots d x_{k+\frac{p}{2}}^{\prime} \\
& \quad \prod_{\ell=k+1}^{k+\frac{p}{2}} \delta\left(x_{j}-x_{\ell}\right) \delta\left(x_{j}-x_{\ell}^{\prime}\right) \gamma^{\left(k+\frac{p}{2}\right)}\left(t, x_{1}, \ldots, x_{k+\frac{p}{2}} ; x_{1}^{\prime}, \ldots, x_{k+\frac{p}{2}}^{\prime}\right)
\end{aligned}
$$

and

$$
\begin{aligned}
& \left(B_{j ; k+1, \ldots, k+\frac{p}{2}}^{-} \gamma^{\left(k+\frac{p}{2}\right)}\right)\left(t, x_{1}, \ldots, x_{k} ; x_{1}^{\prime}, \ldots, x_{k}^{\prime}\right) \\
& \quad=\int d x_{k+1} \cdots d x_{k+\frac{p}{2}} d x_{k+1}^{\prime} \cdots d x_{k+\frac{p}{2}}^{\prime} \\
& \quad \prod_{\ell=k+1}^{k+\frac{p}{2}} \delta\left(x_{j}^{\prime}-x_{\ell}\right) \delta\left(x_{j}^{\prime}-x_{\ell}^{\prime}\right) \gamma^{\left(k+\frac{p}{2}\right)}\left(t, x_{1}, \ldots, x_{k+\frac{p}{2}} ; x_{1}^{\prime}, \ldots, x_{k+\frac{p}{2}}^{\prime}\right) .
\end{aligned}
$$

The operator $B_{k+\frac{p}{2}} \gamma^{\left(k+\frac{p}{2}\right)}$ accounts for $\frac{p}{2}+1$-body interactions between the Bose particles. We note that for factorized solutions, the corresponding 1-particle wave function satisfies the $p$-NLS $i \partial_{t} \phi=-\Delta \phi+\mu|\phi|^{p} \phi$.

Following our conventions in [8, 9, we refer to (2.7) as the cubic GP hierarchy if $p=2$, and as the quintic GP hierarchy if $p=4$. Moreover, we denote the $L^{2}$-critical exponent by $p_{L^{2}}=\frac{4}{d}$, and refer to (2.7) as a: 
- $L^{2}$-critical GP hierarchy if $p=p_{L^{2}}$.

- $L^{2}$-subcritical GP hierarchy if $p<p_{L^{2}}$.

- $L^{2}$-supercritical GP hierarchy if $p>p_{L^{2}}$.

In an analogous manner, we use the notion of energy-critical (respectively, energysubcritical and energy-supercritical GP hierarchies) if $p=p_{H^{1}}$ (respectively, $p<$ $p_{H^{1}}$ and $p>p_{H^{1}}$ ), where $p_{H^{1}}=\frac{4}{d-2}$. Moreover, we respectively refer to the cases $\mu=1$ or $\mu=-1$ as defocusing or focusing GP hierarchies.

For notational brevity, we introduced the following compact notation for the $p$-GP hierarchy in 8 ,

$$
\begin{aligned}
i \partial_{t} \Gamma+\widehat{\Delta}_{ \pm} \Gamma & =\mu \widehat{B} \Gamma \\
\Gamma(0) & =\Gamma_{0}
\end{aligned}
$$

where

$$
\widehat{\Delta}_{ \pm} \Gamma:=\left(\Delta_{ \pm}^{(k)} \gamma^{(k)}\right)_{k \in \mathbb{N}} \quad \text { with } \quad \Delta_{ \pm}^{(k)}=\Delta_{\underline{x}_{k}}-\Delta_{\underline{x}_{k}^{\prime}}
$$

and

$$
\widehat{B} \Gamma:=\left(B_{k+\frac{p}{2}} \gamma^{\left(k+\frac{p}{2}\right)}\right)_{k \in \mathbb{N}} .
$$

Moreover, we will use the notation

$$
\begin{aligned}
\widehat{B}^{+} \Gamma & :=\left(B_{k+\frac{p}{2}}^{+} \gamma^{\left(k+\frac{p}{2}\right)}\right)_{k \in \mathbb{N}}, \\
\widehat{B}^{-} \Gamma & :=\left(B_{k+\frac{p}{2}}^{-} \gamma^{\left(k+\frac{p}{2}\right)}\right)_{k \in \mathbb{N}} .
\end{aligned}
$$

\section{Statement of the main Theorems}

In this section, we state the main results of this paper. In Theorem 3.1, we establish the conservation of novel higher order energy functionals for solutions of $p$-GP hierarchies in $\mathfrak{H}_{\xi}^{1}$, which are first introduced in this paper. This result is used to prove a priori energy bounds on positive semidefinite solutions for defocusing, energy subcritical $p$-GP hierarchies in Theorem 3.2, and for (de)focusing $L^{2}$-subcritical $p$-GP hierarchies in Theorem 3.3 .

We note that the local well-posedness of solutions in the space $\mathfrak{H}_{\xi}^{1}$ has so far been an open problem. While the unconditional uniqueness (that is, without any requirement on the a priori boundedness of some Strichartz norm) of solutions in $\mathfrak{H}_{\xi}^{1}$ has been proven in 11, 12, existence of solutions is only established for factorized data. This is because in [11, 12, solutions to the GP hierarchy have been derived only for initial data for the $N$-body Schrodinger system that are asymptotically factorizing.

Our local well-posedness result proven in $[8$ implies the well-posedness of solutions in $\mathcal{H}_{\xi}^{1}$ with initial data in $\mathfrak{H}_{\xi}^{1} \subset \mathcal{H}_{\xi}^{1}$, under the requirement that $\widehat{B} \Gamma \in L_{t}^{2} \mathcal{H}_{\xi}^{\alpha}$ holds, similar to the a priori space time bound introduced by Klainerman and Machedon in 20]. Using the conserved, higher order energy functionals, we prove in this paper that positive semidefinite solutions of this form remain in $\mathfrak{H}_{\xi}^{1}$, and can in fact be enhanced to global solutions. This is demonstrated for the defocusing energy subcritical case, and for the $L^{2}$-subcritical (de)focusing case. 
Our a priori upper bounds on the $\mathfrak{H}_{\xi}^{1}$-norm of $\Gamma$ hold for positive semidefinite $\Gamma$, as has been noted here and in the introduction. Positive definiteness is physically meaningful because the components of $\Gamma$ are interpreted as density matrices. An obvious example is given by arbitrary linear superpositions of factorized states with positive coefficients, $\Gamma_{0}=\sum \mu_{j} \Gamma_{\phi_{j}}, \mu_{j}>0$, and $\Gamma_{\phi_{j}}=\left(\left(\left|\phi_{j}\right\rangle\left\langle\phi_{j}\right|\right)^{\otimes n}\right)_{n \in \mathbb{N}}$, $\phi_{j} \in H^{1}\left(\mathbb{R}^{d}\right)$. Furthermore, $\Gamma(t)$ obtained from the $N \rightarrow \infty$ limit of the BBGKY hierarchy of an $N$-body Schrodinger equation is generally expected to be positive semidefinite. The question whether $\Gamma(t)$ is positive semidefinite whenever the same holds for $\Gamma_{0}$ is not well understood, as far as we know, and we leave a more systematic study of this issue to future work.

Theorem 3.1. Let $\Gamma(t) \in \mathfrak{H}_{\xi}^{1}$ be a solution of the $p$-GP hierarchy, for $t \in I \subseteq \mathbb{R}$ with $\{0\} \in I$, and some $0<\xi<1$. Then, the infinite family of operators $\left(\mathcal{K}^{(m)}\right)_{m \in \mathbb{N}}$ presented in (4.3) below define an infinite sequence of linear functionals,

$$
\Gamma(t) \mapsto\left(\left\langle\mathcal{K}^{(m)}\right\rangle_{\Gamma(t)}\right)_{m \in \mathbb{N}}
$$

where

$$
\left\langle\mathcal{K}^{(m)}\right\rangle_{\Gamma(t)}:=\operatorname{Tr}\left(\mathcal{K}^{(m)} \gamma^{\left(m k_{p}\right)}(t)\right),
$$

which we refer to as higher order energy functionals. Here, we recall that $\Gamma(t)=\left(\gamma^{(n)}(t)\right)_{n \in \mathbb{N}}$ and $k_{p}=1+\frac{p}{2}$. The higher order energy functionals have the following properties:

- They are bounded, and in particular, there exists $0<\hat{\xi}<\xi$ such that

$$
\sum_{m \in \mathbb{N}} \hat{\xi}^{m}\left\langle\mathcal{K}^{(m)}\right\rangle_{\Gamma(t)} \leq\|\Gamma(t)\|_{\mathfrak{H}_{\xi}^{1}}
$$

holds, for all $t \in I$.

- The higher order energy functionals are conserved,

$$
\left\langle\mathcal{K}^{(m)}\right\rangle_{\Gamma(t)}=\left\langle\mathcal{K}^{(m)}\right\rangle_{\Gamma(0)}
$$

for all $t \in I$, and all $m \in \mathbb{N}$.

- Assuming that the initial condition satisfies $\Gamma(0) \in \mathfrak{H}_{\xi^{\prime}}^{1}$ for some $0<\xi^{\prime}<1$, and that

$$
\xi \leq\left(1+\frac{2}{p+2} C_{S o b}(d, p)\right)^{-\frac{1}{k_{p}}} \xi^{\prime}
$$

where the constant $C_{S o b}(d, p)$ is as in Theorem 5.1 below, the a priori bound

$$
\sum_{m \in \mathbb{N}}(2 \xi)^{m}\left\langle\mathcal{K}^{(m)}\right\rangle_{\Gamma(t)} \leq\left\|\Gamma_{0}\right\|_{\mathfrak{H}_{\xi^{\prime}}^{1}}
$$

is satisfied for $p<\frac{4}{d-2}$ and $|\mu| \leq 1$ (focusing and defocusing hierarchies).

Accordingly, the higher order energy functionals are bounded, and for sufficiently small $\xi>0$, the associated power series (3.6) is bounded by the initial data of the solution $\Gamma(t) \in \mathfrak{H}_{\xi}^{1}$, due to their time invariance.

On the other hand, the higher order energy functionals provide us with a priori bounds on the $\mathfrak{H}_{\xi}^{1}$ norm of the solution itself. In case of defocusing $p$-GP hierarchies, we find the following result. 
Theorem 3.2. Assume that $\mu=+1$ (defocusing $p$-GP hierarchy), $p<\frac{4}{d-2}$, and that $\Gamma(t) \in \mathfrak{H}_{\xi}^{1}$ for $t \in[0, T]$, is a positive semidefinite solution of the $p$-GP hierarchy with initial data $\Gamma_{0} \in \mathfrak{H}_{\xi^{\prime}}^{1}$ for $\xi, \xi^{\prime}$ as in (3.5). Then, the a priori bound

$$
\begin{aligned}
\|\Gamma(t)\|_{\mathfrak{H}_{\xi}^{1}} & \leq \sum_{m \in \mathbb{N}}(2 \xi)^{m}\left\langle\mathcal{K}^{(m)}\right\rangle_{\Gamma(t)} \\
& =\sum_{m \in \mathbb{N}}(2 \xi)^{m}\left\langle\mathcal{K}^{(m)}\right\rangle_{\Gamma_{0}} \leq\left\|\Gamma_{0}\right\|_{\mathfrak{H}_{\xi^{\prime}}^{1}}
\end{aligned}
$$

is satisfied for all $t \in[0, T]$.

For focusing, $L^{2}$-subcritical $p$-GP hierarchies, we obtain a similar result, provided that the interaction is not too large.

Theorem 3.3. Let $p<p_{L^{2}}=\frac{4}{d}\left(L^{2}\right.$ subcritical). Moreover, let $\alpha>\alpha_{0}:=\frac{\left(k_{p}-1\right) d}{2 k_{p}}$ and $\alpha k_{p}<1$, where $k_{p}=1+\frac{p}{2}$, and $\alpha<1$. Let

$$
D:=D(\alpha, p, d,|\mu|)=\left(1-|\mu| \frac{C_{0}(\alpha)}{1-4^{-\left(1-\alpha k_{p}\right)}}\right),
$$

where $C_{0}(\alpha)$ is characterized in (6.20).

Assume that $\Gamma(t) \in \mathfrak{H}_{\xi}^{1}$ is a positive semidefinite solution of the focusing $(\mu<0)$ $p$-GP hierarchy for $t \in I$, with initial data $\Gamma(0)=\Gamma_{0} \in \mathfrak{H}_{\xi^{\prime}}^{1}$, where

$$
\xi \leq \frac{1}{D}\left(1+\frac{2}{p+2} C_{S o b}(d, p)\right)^{-\frac{1}{k_{p}}} \xi^{\prime}
$$

If $\mu<0$ is such that

$$
|\mu|<\frac{1-4^{-\left(1-\alpha k_{p}\right)}}{C_{0}(\alpha)}
$$

then the a priori bound

holds for all $t \in I$.

$$
\begin{aligned}
\|\Gamma(t)\|_{\mathfrak{H}_{\xi}^{1}} & \leq \sum_{m=1}^{\infty}(2 D \xi)^{m}\left\langle\mathcal{K}^{(m)}\right\rangle_{\Gamma(t)} \\
& =\sum_{m=1}^{\infty}(2 D \xi)^{m}\left\langle\mathcal{K}^{(m)}\right\rangle_{\Gamma_{0}} \\
& \leq\left\|\Gamma_{0}\right\|_{\mathfrak{H}_{\xi^{\prime}}^{1}}
\end{aligned}
$$

In [8], we proved local well-posedness of solutions in the spaces $\Gamma \in L_{t}^{\infty} \mathcal{H}_{\xi}^{\alpha}$ under the condition that $\widehat{B} \Gamma \in L_{t}^{2} \mathcal{H}_{\xi}^{\alpha}$.

Theorem 3.4. Let

$$
\alpha \in \mathfrak{A}(d, p):=\left\{\begin{array}{cc}
\left(\frac{1}{2}, \infty\right) & \text { if } d=1 \\
\left(\frac{d}{2}-\frac{1}{2(p-1)}, \infty\right) & \text { if } d \geq 2 \text { and }(d, p) \neq(3,2) \\
{[1, \infty)} & \text { if }(d, p)=(3,2) .
\end{array}\right.
$$

Then, there exists a constant $0<\eta<1$ such that for $0<\xi \leq \eta \xi^{\prime} \leq 1$, there exists a constant $T_{0}\left(d, p, \xi, \xi^{\prime}\right)>0$ such that the following holds. Let $I:=[0, T]$ 
for $0<T<T_{0}\left(d, p, \xi, \xi^{\prime}\right)$. Then, there exists a unique solution $\Gamma \in L_{t \in I}^{\infty} \mathcal{H}_{\xi}^{\alpha}$ of the p-GP hierarchy, with

$$
\|\widehat{B} \Gamma\|_{L_{t \in I}^{1} \mathcal{H}_{\xi}^{\alpha}}<C\left(T, \xi, \xi^{\prime}, d, p\right)\left\|\Gamma_{0}\right\|_{\mathcal{H}_{\xi^{\prime}}^{\alpha}},
$$

in the space

$$
\mathcal{W}^{\alpha}(I, \xi)=\left\{\Gamma \in L_{t \in I}^{\infty} \mathcal{H}_{\xi}^{\alpha} \mid \widehat{B}^{+} \Gamma, \widehat{B}^{-} \Gamma \in L_{t \in I}^{2} \mathcal{H}_{\xi}^{\alpha}\right\}
$$

for the initial condition $\Gamma(0)=\Gamma_{0} \in \mathcal{H}_{\xi^{\prime}}^{\alpha}$.

We note that the presence of two different energy scales $\xi, \xi^{\prime}$ has the following interpretation on the level of the NLS. Let $R_{0}:=\left(\xi^{\prime}\right)^{-1 / 2}$ and $R_{1}:=\xi^{-1 / 2}$. Then, the local well-posedness result in Theorem 3.4 applied to factorized initial data $\Gamma_{0}=\Gamma_{\phi_{0}}$, is equivalent to the following statement: For $\left\|\phi_{0}\right\|_{H^{1}\left(\mathbb{R}^{n}\right)}<R_{0}$, there exists a unique solution $\|\phi\|_{L_{t \in I}^{\infty} H^{1}\left(\mathbb{R}^{n}\right)}<R_{1}$, with $R_{1}>R_{0}$, in the space

$$
\left\{\phi \in L_{t \in I}^{\infty} H^{1}\left(\mathbb{R}^{n}\right)\left|\left\||\phi|^{p} \phi\right\|_{L_{t}^{2} H^{1}}<\infty\right\} .\right.
$$

This version of local well-posedness, specified for balls $B_{R_{0}}(0), B_{R_{1}}(0) \subset H^{1}\left(\mathbb{R}^{n}\right)$, contains the less specific formulation of local well-posedness where only finiteness is required, $\left\|\phi_{0}\right\|_{H^{1}\left(\mathbb{R}^{n}\right)}<\infty$ and $\|\phi\|_{L_{t \in I}^{\infty} H^{1}\left(\mathbb{R}^{n}\right)}<\infty$.

In this paper we enhance local to global wellposedness for solutions in the spaces $\mathcal{H}_{\xi}^{1}$ constructed in [8], for initial data in $\mathfrak{H}_{\xi^{\prime}}^{1} \subset \mathcal{H}_{\xi^{\prime}}^{1}$ with $\xi<\xi^{\prime}$, provided that the solution is positive semidefinite. The proof is again based on the use of the higher order energy functionals.

Theorem 3.5. Assume one of the following two cases:

- Energy subcritical, defocusing $p$-GP hierarchy with $p<\frac{4}{d-2}$ and $\mu=+1$. Moreover, $\xi$, $\xi^{\prime}$ satisfy (3.5).

- $L^{2}$ subcritical, focusing $p$-GP hierarchy with $p<\frac{4}{d}$ and $\mu<0$ satisfying (3.10). In addition, $\xi$, $\xi^{\prime}$ satisfy (3.9).

Then, there exists $T>0$ such that for $I_{j}:=[j T,(j+1) T]$, with $j \in \mathbb{Z}$, there exists a unique global solution $\Gamma \in \cup_{j \in \mathbb{Z}} \mathcal{W}^{1}\left(I_{j}, \xi\right)$ of the $p$-GP hierarchy with initial condition $\Gamma(0)=\Gamma_{0} \in \mathcal{H}_{\xi^{\prime}}^{1}$, satisfying

$$
\|\Gamma(t)\|_{\mathcal{H}_{\xi}^{1}} \leq\|\Gamma(t)\|_{\mathfrak{H}_{\xi}^{1}} \leq C\left\|\Gamma_{0}\right\|_{\mathfrak{H}_{\xi^{\prime}}^{1}}
$$

for all $t \in \mathbb{R}$, if $\Gamma(t)$ is positive semidefinite for all $t \in I_{j}, j \in \mathbb{Z}$.

\section{Higher order ENERGy CONSERVATion}

In this section, we introduce a higher order generalization of the energy functional introduced in $[9$. We prove that it is a conserved quantity for solutions of the $p$-GP hierarchy. As a main application, this conserved quantity will be used to enhance local well-posedness (obtained in [8]) to global well-posedness for certain defocusing GP-hierarchies in the spaces $\mathcal{H}_{\xi}^{1}$, for initial data in $\mathfrak{H}_{\xi}^{1}$ (see Section 7 for details).

Let

$$
k_{p}:=1+\frac{p}{2} .
$$


We define the operators

$$
K_{\ell}:=\frac{1}{2}\left(1-\Delta_{x_{\ell}}\right) \operatorname{Tr}_{\ell+1, \ldots, \ell+\frac{p}{2}}+\frac{\mu}{p+2} B_{\ell ; \ell+1, \ldots, \ell+\frac{p}{2}}^{+}
$$

for $\ell \in \mathbb{N}$. This operator is related to the average energy per particle $E_{1}(\Gamma)$ (introduced in [9]) through

$$
\begin{aligned}
\frac{1}{2} & +E_{1}(\Gamma) \\
& =\operatorname{Tr}_{1, \ldots, \ell, \ell+k, \cdots, j} K_{\ell} \gamma^{(j)} \\
& =\frac{1}{2}+\frac{1}{2} \operatorname{Tr}_{1}\left(-\Delta_{x} \gamma^{(1)}\right)+\frac{\mu}{p+2} \int d x \gamma^{\left(k_{p}\right)}(x, \ldots, x ; x, \ldots, x),
\end{aligned}
$$

using the admissibility of $\Gamma=\left(\gamma^{j}\right)_{j \in \mathbb{N}}$, see also $[9]$.

Moreover, we introduce the operator

$$
\mathcal{K}^{(m)}:=K_{1} K_{k_{p}+1} \cdots K_{(m-1) k_{p}+1}
$$

where the $m$ factors are mutually commuting, in the sense that

$$
K_{j k_{p}+1} K_{j^{\prime} k_{p}+1} \gamma^{(j)}=K_{j^{\prime} k_{p}+1} K_{j k_{p}+1} \gamma^{(j)}
$$

holds for $0 \leq j \neq j^{\prime} \leq m-1$.

We may now give the precise statement of our main result that provides the conservation of higher energy functional for solutions of the $p$-GP hierarchy.

Theorem 4.1. Assume that $\Gamma=\left(\gamma^{(j)}\right) \in \mathfrak{H}_{\xi}^{1}$ is admissible and solves the $p$-GP hierarchy. Then, for all $m \in \mathbb{N}$, the higher order energy functionals

$$
\left\langle\mathcal{K}^{(m)}\right\rangle_{\Gamma(t)}:=\operatorname{Tr}_{1, k_{p}+1,2 k_{p}+1, \ldots,(m-1) k_{p}+1}\left(\mathcal{K}^{(m)} \gamma^{\left(m k_{p}\right)}(t)\right)
$$

are bounded, and are conserved quantities, i.e.

$$
\partial_{t}\left\langle\mathcal{K}^{(m)}\right\rangle_{\Gamma(t)}=0
$$

for every $m \in \mathbb{N}$.

Remark 4.2. As is shown in Theorem [6.1 below, $\left\langle\mathcal{K}^{(m)}\right\rangle_{\Gamma(t)}$ are bounded, and in particular, there exists $0<\hat{\xi}<\xi$ such that

$$
\sum_{m \in \mathbb{N}} \hat{\xi}^{m}\left\langle\mathcal{K}^{(m)}\right\rangle_{\Gamma(t)} \leq\|\Gamma(t)\|_{\mathfrak{H}_{\xi}^{1}}
$$

holds, for all $t \in I$. Hence, the conserved quantities $\left\langle\mathcal{K}^{(m)}\right\rangle_{\Gamma(t)}$ are well defined for solutions of the GP hierarchy $\Gamma=\left(\gamma^{(j)}\right) \in \mathfrak{H}_{\xi}^{1}$.

Remark 4.3. We note that in the definition of $\left\langle\mathcal{K}^{(m)}\right\rangle_{\Gamma(t)}$, we may replace $\gamma^{\left(m k_{p}\right)}$ by any $\gamma^{(j)}$ with $j \geq m k_{p}$, and would still obtain the same value of $\left\langle\mathcal{K}^{(m)}\right\rangle_{\Gamma(t)}$.

Proof. To prove (4.6), we note that

$$
i \partial_{t} \gamma^{\left(m k_{p}\right)}=\sum_{\ell=1}^{m}\left(h_{\ell}^{ \pm} \gamma^{\left(m k_{p}\right)}+\mu b_{\ell}^{ \pm} \gamma^{\left(m k_{p}+\frac{p}{2}\right)}\right)
$$


where

$$
\begin{aligned}
h_{\ell}^{ \pm} \gamma^{\left(m k_{p}\right)} & \left(\underline{x}_{m k_{p}} ; \underline{x}_{m k_{p}}^{\prime}\right) \\
& :=-\sum_{j=(\ell-1) k_{p}+1}^{\ell k_{p}}\left(\Delta_{x_{j}}-\Delta_{x_{j}^{\prime}}\right) \gamma^{\left(m k_{p}\right)}\left(\underline{x}_{m k_{p}} ; \underline{x}_{m k_{p}}^{\prime}\right)
\end{aligned}
$$

and

$$
\begin{aligned}
& \left(b_{\ell}^{ \pm} \gamma^{\left(m k_{p}+\frac{p}{2}\right)}\right)\left(\underline{x}_{m k_{p}} ; \underline{x}_{m k_{p}}^{\prime}\right) \\
& \quad:=\sum_{j=(\ell-1) k_{p}+1}^{\ell k_{p}}\left(B_{j ; m k_{p}+1, \ldots, m k_{p}+\frac{p}{2}}^{ \pm} \gamma^{\left(m k_{p}+\frac{p}{2}\right)}\right)\left(\underline{x}_{m k_{p}} ; \underline{x}_{m k_{p}}^{\prime}\right)
\end{aligned}
$$

Accordingly,

$$
\partial_{t}\left\langle\mathcal{K}^{(m)}\right\rangle_{\Gamma(t)}=\sum_{\ell=1}^{m}[A(\ell ; m)]
$$

where

$$
\begin{aligned}
& A(\ell ; m) \\
& \quad:=\operatorname{Tr}_{1, k_{p}+1,2 k_{p}+1, \ldots,(m-1) k_{p}+1}\left(\mathcal{K}^{(m)}\left(h_{\ell}^{ \pm} \gamma^{\left(m k_{p}\right)}+\mu b_{\ell}^{ \pm} \gamma^{\left(m k_{p}+\frac{p}{2}\right)}\right)\right) .
\end{aligned}
$$

We claim that

$$
A(\ell ; m)=0
$$

for every $\ell \in\{1, \ldots, m\}$.

To prove this, we first of all note that by symmetry of $\gamma^{\left(m k_{p}\right)}\left(\underline{x}_{m k_{p}} ; \underline{x}_{m k_{p}}^{\prime}\right)$ with respect to the components of $\underline{x}_{m k_{p}}$ and $\underline{x}_{m k_{p}}^{\prime}$, it suffices to assume that $\ell=1$. The other cases are similar.

Accordingly, letting $\ell=1$, we introduce the notations

$$
\widetilde{\gamma}^{\left(k_{p}\right)}\left(\underline{x}_{k_{p}} ; \underline{x}_{k_{p}}^{\prime}\right):=\operatorname{Tr}_{k_{p}+1, \ldots, m k_{p}}\left(K_{k_{p}+1} \cdots K_{(m-1) k_{p}+1} \gamma^{\left(m k_{p}\right)}\right)
$$

and

$$
\begin{aligned}
& \widetilde{\gamma}^{\left(2 k_{p}-1\right)}\left(\underline{x}_{k_{p}}, \underline{y}_{k_{p}-1} ; \underline{x}_{k_{p}}^{\prime}, \underline{y}_{k_{p}-1}^{\prime}\right) \\
& \quad:=\operatorname{Tr}_{k_{p}+1, \ldots, m k_{p}}\left(K_{k_{p}+1} \cdots K_{(m-1) k_{p}+1} \gamma^{\left(m k_{p}+\frac{p}{2}\right)}\right)\left(\underline{x}_{k_{p}}, \underline{y}_{k_{p}-1} ; \underline{x}_{k_{p}}^{\prime}, \underline{y}_{k_{p}-1}^{\prime}\right)
\end{aligned}
$$

where $y_{i}=x_{m k_{p}+i}$ and $y_{i}^{\prime}=x_{m k_{p}+i}^{\prime}$, for $i \in\left\{1, \ldots, k_{p}-1\right\}$.

In what follows, we will keep $m$ fixed, and omit it from the notation. Given $\widetilde{\gamma}^{(j)}$ with $j \in\left\{k_{p}, 2 k_{p}-1\right\}$, we decompose

$$
\mathbb{R}^{j d}=\bigcup_{r \in \mathbb{Z}^{j d}} Q_{r}
$$

into disjoint cubes $Q_{r}$ obtained from translating the unit cube $[0,1)^{j d}$ by $r \in \mathbb{Z}^{j d}$. We then define

$$
\widetilde{\gamma}_{r, r^{\prime}}^{(j)}:=P_{Q_{r}, Q_{r^{\prime}}}\left(\widetilde{\gamma}^{(j)}\right)
$$


where $P_{Q_{r}, Q_{r^{\prime}}}$ is the Fourier multiplication operator with symbol given by the characteristic function of $Q_{r} \times Q_{r^{\prime}}$. That is,

$$
\widehat{\widetilde{\gamma}_{r, r^{\prime}}^{(j)}}\left(\underline{u}_{j} ; \underline{u}_{j}^{\prime}\right)=\chi_{Q_{r}}\left(\underline{u}_{j}\right) \chi_{Q_{r^{\prime}}}\left(\underline{u}_{j}^{\prime}\right) \widehat{\widetilde{\gamma}(j)}\left(\underline{u}_{j} ; \underline{u}_{j}^{\prime}\right)
$$

for $r, r^{\prime} \in \mathbb{Z}^{j d}$.

Using the notation

$$
K_{1}=K_{1}^{(1)}+K_{1}^{(2)}
$$

where

$$
K_{1}^{(1)}:=\frac{1}{2}\left(1-\Delta_{x_{1}}\right) \operatorname{Tr}_{2, \ldots, k_{p}}
$$

and

$$
K_{1}^{(2)}:=\frac{\mu}{p+2} B_{1 ; 2, \ldots, k_{p}}^{+}
$$

we consider

$$
\begin{aligned}
A_{h}^{(1)}\left(r, r^{\prime}\right):= & \operatorname{Tr}_{1}\left(K_{1}^{(1)} h_{1}^{ \pm} \widetilde{\gamma}_{r, r^{\prime}}^{\left(k_{p}\right)}\right) \\
= & -\frac{1}{2} \operatorname{Tr}_{1,2, \ldots, k_{p}}\left(\left(1-\Delta_{x_{1}}\right) \sum_{j=1}^{k_{p}}\left(\Delta_{x_{j}}-\Delta_{x_{j}^{\prime}}\right) \widetilde{\gamma}_{r, r^{\prime}}^{\left(k_{p}\right)}\right) \\
= & \frac{1}{2} \int d u_{1} \ldots d u_{k_{p}} d u_{1}^{\prime} \ldots d u_{k_{p}}^{\prime} \int d x_{1} \ldots d x_{k_{p}} d x_{1}^{\prime} \ldots d x_{k_{p}}^{\prime} \\
& \delta\left(x_{1}-x_{1}^{\prime}\right) \ldots \delta\left(x_{k_{p}}-x_{k_{p}}^{\prime}\right)\left(1+u_{1}^{2}\right) \sum_{j=1}^{k_{p}}\left(u_{j}^{2}-\left(u_{j}^{\prime}\right)^{2}\right) \\
= & \frac{1}{2} \int_{Q_{r} \times Q_{r^{\prime}}} \quad\left(\prod_{l=1}^{k_{p}} e^{i\left(u_{l} x_{l}-u_{l}^{\prime} x_{l}^{\prime}\right)} \widehat{\widehat{\widetilde{\gamma}_{r, r^{\prime}}\left(k_{p^{\prime}}\right)}}\left(\underline{u}_{k_{p}} ; \underline{u}_{k_{p}}^{\prime}\right)\right. \\
& \left(1+u_{k_{p}} d u_{1}^{\prime} \ldots d u_{k_{p}}^{\prime}\right) \sum_{j=1}^{k_{p}}\left(\prod_{l=1}^{k_{p}} \delta\left(u_{j}^{2}-\left(u_{j}^{\prime}\right)^{2}\right) \widehat{\widetilde{\gamma}^{\left(k_{p}\right)}}\left(\underline{u}_{k_{p}} ; \underline{u}_{k_{p}}^{\prime}\right)\right)
\end{aligned}
$$

for $Q_{r}, Q_{r^{\prime}} \in \mathbb{R}^{d k_{p}}$ and $r, r^{\prime} \in \mathbb{Z}^{d k_{p}}$.

Moreover, for $Q_{r}, Q_{r^{\prime}} \in \mathbb{R}^{d\left(2 k_{p}-1\right)}$ and $r, r^{\prime} \in \mathbb{Z}^{d\left(2 k_{p}-1\right)}$, we let

$$
A_{b}^{(1)}\left(r, r^{\prime}\right):=\operatorname{Tr}_{1}\left(K_{1}^{(1)} b_{1}^{ \pm} \widetilde{\gamma}_{r, r^{\prime}}^{\left(2 k_{p}-1\right)}\right)
$$

and

$$
\begin{aligned}
A_{h}^{(2)}\left(r, r^{\prime}\right) & :=\operatorname{Tr}_{1}\left(K_{1}^{(2)} h_{1}^{ \pm} \widetilde{\gamma}_{r, r^{\prime}}^{\left(k_{p}\right)}\right) \\
& =\operatorname{Tr}_{1}\left(K_{1}^{(2)} h_{1}^{ \pm} \operatorname{Tr}_{k_{p}+1 \cdots 2 k_{p}-1} \widetilde{\gamma}_{r, r^{\prime}}^{\left(2 k_{p}-1\right)}\right)
\end{aligned}
$$


where we used admissibility to pass to the last line. Also we let

$$
\begin{aligned}
A_{b}^{(2)}\left(r, r^{\prime}\right) & :=\operatorname{Tr}_{1}\left(K_{1}^{(2)} b_{1}^{ \pm} \widetilde{\gamma}_{r, r^{\prime}}^{\left(2 k_{p}-1\right)}\right) \\
& =\frac{\mu}{p+2} \operatorname{Tr}_{1}\left(B_{1 ; 2, \ldots, k_{p}}^{+} b_{1}^{ \pm} \widetilde{\gamma}_{r, r^{\prime}}^{\left(2 k_{p}-1\right)}\right) \\
& =\frac{\mu}{p+2} \sum_{j=1}^{k_{p}} \operatorname{Tr}_{1}\left(B_{1 ; 2, \ldots, k_{p}}^{+} B_{j ; k_{p}+1, \ldots, 2 k_{p}-1}^{ \pm} \widetilde{\gamma}_{r, r^{\prime}}^{\left(2 k_{p}-1\right)}\right) \\
& =\frac{\mu}{p+2} \sum_{j=1}^{k_{p}} \int d x_{1} \ldots d x_{k_{p}} d x_{1}^{\prime} \ldots d x_{k_{p}}^{\prime} \delta\left(x_{1}=\cdots=x_{k_{p}}=x_{1}^{\prime}=\cdots=x_{k_{p}}^{\prime}\right) \\
& \left(B_{j ; k_{p}+1, \ldots, 2 k_{p}-1}^{ \pm} \widetilde{\gamma}_{r, r^{\prime}}^{\left(2 k_{r^{\prime}}-1\right)}\right)\left(\underline{x}_{k_{p}}, \underline{x}_{k_{p}}^{\prime}\right)
\end{aligned}
$$

where we have adopted the notation

$$
\begin{aligned}
\delta\left(x_{1}=\cdots=x_{k_{p}}=x_{1}^{\prime}=\cdots=x_{k_{p}}^{\prime}\right) \\
\quad:=\delta\left(x_{1}-x_{1}^{\prime}\right) \prod_{\ell=2}^{k_{p}}\left(\delta\left(x_{1}-x_{\ell}\right) \delta\left(x_{1}-x_{\ell}^{\prime}\right)\right)
\end{aligned}
$$

from [9].

Proof of $A_{h}^{(1)}\left(r, r^{\prime}\right)=0$. We recall that $Q_{r} \in \mathbb{R}^{d k_{p}}$ and $r, r^{\prime} \in \mathbb{Z}^{d k_{p}}$. We have

$$
\begin{aligned}
& A_{h,+}^{(1)}\left(r, r^{\prime}\right):= \frac{1}{2} \int_{Q_{r} \times Q_{r^{\prime}}} d u_{1} \ldots d u_{k_{p}} d u_{1}^{\prime} \ldots d u_{k_{p}}^{\prime}\left(\prod_{l=1}^{k_{p}} \delta\left(u_{l}-u_{l}^{\prime}\right)\right) \\
&\left(1+u_{1}^{2}\right) \sum_{j=1}^{k_{p}} u_{j}^{2} \widehat{\widehat{\gamma}^{\left(k_{p}\right)}}\left(\underline{u}_{k_{p}} ; \underline{u}_{k_{p}}^{\prime}\right) \\
&=\delta_{r, r^{\prime}} \frac{1}{2} \int_{Q_{r}} d u_{1} \ldots d u_{k_{p}}\left(1+u_{1}^{2}\right)\left(\sum_{j=1}^{k_{p}} u_{j}^{2}\right) \widehat{\widetilde{\gamma}^{\left(k_{p}\right)}}\left(\underline{u}_{k_{p}} ; \underline{u}_{k_{p}}\right) \\
& \leq \sup _{\underline{u}_{k_{p}} \in Q_{r}}\left(\frac{1}{2}\left(1+u_{1}^{2}\right)\left(\sum_{j=1}^{k_{p}} u_{j}^{2}\right)\right) \operatorname{Tr}\left(\widetilde{\gamma}^{\left(k_{p}\right)}\right) \\
& \leq C\left(Q_{r}\right)
\end{aligned}
$$

is finite, for every choice of $Q_{r}$. Therefore,

$$
A_{h}^{(1)}\left(r, r^{\prime}\right)=A_{h,+}^{(1)}\left(r, r^{\prime}\right)-A_{h,-}^{(1)}\left(r, r^{\prime}\right)=0,
$$

as claimed, since $A_{h,-}^{(1)}\left(r, r^{\prime}\right)=A_{h,+}^{(1)}\left(r, r^{\prime}\right)$ from direct comparison of terms in (4.25) and (4.22).

Proof of $A_{b}^{(2)}\left(r, r^{\prime}\right)=0$. We recall that in this situation, we have $Q_{r} \in \mathbb{R}^{d\left(2 k_{p}-1\right)}$ and $r, r^{\prime} \in \mathbb{Z}^{d\left(2 k_{p}-1\right)}$. Using the definition of $B_{j ; k_{p}+1, \ldots, 2 k_{p}-1}^{ \pm}$, we find that the term 
in $A_{b}^{(2)}\left(r, r^{\prime}\right)$ involving $B_{j ; k_{p}+1, \ldots, 2 k_{p}-1}^{+}$is given by

$$
\begin{gathered}
A_{b,+}^{(2)}\left(r, r^{\prime}\right) \\
=\frac{\mu}{p+2} \sum_{j=1}^{k_{p}} \int d x_{1} \ldots d x_{k_{p}} d x_{1}^{\prime} \ldots d x_{k_{p}}^{\prime} \delta\left(x_{1}=\ldots=x_{k_{p}}=x_{1}^{\prime}=\cdots=x_{k_{p}}^{\prime}\right) \\
\widetilde{\gamma}_{r, r^{\prime}}^{\left(2 k_{p}-1\right)}(x_{1}, \ldots, x_{k_{p}}, \underbrace{x_{j}, \ldots, x_{j}}_{k_{p}-1} ; x_{1}^{\prime}, \ldots, x_{k_{p}}^{\prime}, \underbrace{x_{j}, \ldots, x_{j}}_{k_{p}-1}) \\
=\frac{\mu k_{p}}{p+2} \int_{Q_{r} \times Q_{r^{\prime}}} d u_{1} \ldots d u_{2 k_{p}-1} d u_{1}^{\prime} \ldots d u_{2 k_{p}-1}^{\prime} \delta\left(\sum_{j=1}^{2 k_{p}-1}\left(u_{j}-u_{j}^{\prime}\right)\right) \\
\left.\widetilde{\gamma}^{\left(2 k_{p}-1\right.}\right)\left(\underline{u}_{2 k_{p}-1} ; \underline{u}_{2 k_{p}-1}^{\prime}\right) .
\end{gathered}
$$

We need to verify that it is well-defined.

To this end, we write

$$
\widetilde{\gamma}^{\left(2 k_{p}-1\right)}\left(s ; \underline{u}_{2 k_{p}-1} ; \underline{u}_{2 k_{p}-1}^{\prime}\right)=\sum_{j} \lambda_{j}(s) \phi_{j}\left(\underline{u}_{2 k_{p}-1}\right) \overline{\phi_{j}\left(\underline{u}_{2 k_{p}-1}^{\prime}\right)},
$$

where $\left\{\phi_{j}\right\}$ is an orthonormal basis of $L^{2}\left(\mathbb{R}^{d\left(2 k_{p}-1\right)}\right)$, and $\sum_{j} \lambda_{j}(s)=1$, with $0 \leq \lambda_{j}(s) \leq 1$. We observe that

$$
\begin{aligned}
& \left|\int_{Q_{r} \times Q_{r^{\prime}}} d \underline{u}_{2 k_{p}-1} d \underline{u}_{2 k_{p}-1}^{\prime} \delta\left(\sum_{i=1}^{2 k_{p}-1}\left(u_{i}-u_{i}^{\prime}\right)\right) \phi_{j}\left(\underline{u}_{2 k_{p}-1}\right) \overline{\phi_{j}\left(\underline{u}_{2 k_{p}-1}^{\prime}\right)}\right| \\
& \quad \leq \frac{1}{2} \int_{Q_{r} \times Q_{r^{\prime}}} d \underline{u}_{2 k_{p}-1} d \underline{u}_{2 k_{p}-1}^{\prime} \delta\left(\sum_{i=1}^{2 k_{p}-1}\left(u_{i}-u_{i}^{\prime}\right)\right)\left(\left|\phi_{j}\left(\underline{u}_{2 k_{p}-1}\right)\right|^{2}+\left|\phi_{j}\left(\underline{u}_{2 k_{p}-1}^{\prime}\right)\right|^{2}\right) \\
& =: \frac{1}{2}((i)+(i i)),
\end{aligned}
$$

where

$$
\begin{aligned}
(i) & =\int_{Q_{r} \times Q_{r^{\prime}}} d \underline{u}_{2 k_{p}-1} d \underline{u}_{2 k_{p}-1}^{\prime} \delta\left(\sum_{i=1}^{2 k_{p}-1}\left(u_{i}-u_{i}^{\prime}\right)\right)\left|\phi_{j}\left(\underline{u}_{2 k_{p}}\right)\right|^{2} \\
& =\int_{Q_{r}} d \underline{u}_{2 k_{p}-1}\left|\phi_{j}\left(\underline{u}_{2 k_{p}-1}\right)\right|^{2} A\left(\sum_{i=1}^{2 k_{p}-1} u_{i}\right) \\
& \leq \operatorname{Tr}\left(\widetilde{\gamma}_{r, r}^{\left(2 k_{p}-1\right)}\right) \sup _{\zeta \in \mathbb{R}^{d}} A(\zeta),
\end{aligned}
$$

with

$$
A(\zeta):=\int_{Q_{r^{\prime}}} d \underline{u}_{2 k_{p}-1}^{\prime} \delta\left(\zeta-\sum_{i=1}^{2 k_{p}-1} u_{i}^{\prime}\right) .
$$

We observe that this is the measure of the intersection $Q_{r^{\prime}} \cap L_{\zeta}$ where

$$
L_{\zeta}:=\left\{\underline{u}_{2 k_{p}-1}^{\prime} \in \mathbb{R}^{d\left(2 k_{p}-1\right)} \mid \sum_{i=1}^{2 k_{p}-1} u_{i}^{\prime}=\zeta\right\}
$$


is an affine subspace of dimension $d\left(2 k_{p}-2\right)$, for $\zeta \in \mathbb{R}^{d}$. Clearly,

$$
\operatorname{meas}\left(Q_{r^{\prime}} \cap L_{\zeta}\right)<\operatorname{meas}\left(B_{R} \cap L_{\zeta}\right)<C_{0}
$$

uniformly in $\zeta$, where we may take $B_{R}$ to be the smallest closed ball (with $R$ denoting its radius) concentric with $Q_{r}$ such that $B_{R} \supset Q_{r}$. Indeed, the maximum of meas $\left(B_{R} \cap L_{\zeta}\right)$ is attained when $\zeta$ is such that the center of $B_{R}$ is contained in $L_{\zeta}$, and corresponds to the volume of the $d\left(2 k_{p}-2\right)$-dimensional ball of radius $R$. We conclude that

$$
\begin{aligned}
\sum_{j} \lambda_{j}(s)\left\|\phi_{j}\right\|_{L^{2}}^{2} \sup _{\zeta} A(\zeta) & \leq C_{0} \sum_{j} \lambda_{j}(s) \\
& =C_{0},
\end{aligned}
$$

since $\left\|\phi_{j}\right\|_{L^{2}}^{2}=1$ for all $j$. The same bound holds for the term $(i i)$. This implies that

$$
\left|A_{b,+}^{(2)}\left(r, r^{\prime}\right)\right|<C\left(\operatorname{Tr}\left(\widetilde{\gamma}_{r, r}^{\left(2 k_{p}-1\right)}\right)+\operatorname{Tr}\left(\widetilde{\gamma}_{r^{\prime}, r^{\prime}}^{\left(2 k_{p}-1\right)}\right)\right)<C^{\prime}
$$

Moreover, one can straightforwardly see that including the term in $A_{b}^{(2)}\left(r, r^{\prime}\right)$ involving the operator $B_{j ; k_{p}+1, \ldots, 2 k_{p}-1}^{-}$, one has $A_{b}^{(2)}\left(r, r^{\prime}\right)=A_{b,+}^{(2)}\left(r, r^{\prime}\right)-A_{b,-}^{(2)}\left(r, r^{\prime}\right)$ where $A_{b,-}^{(2)}\left(r, r^{\prime}\right)=A_{b,+}^{(2)}\left(r, r^{\prime}\right)$. Therefore,

$$
A_{b}^{(2)}\left(r, r^{\prime}\right)=A_{b,+}^{(2)}\left(r, r^{\prime}\right)-A_{b,-}^{(2)}\left(r, r^{\prime}\right)=0
$$

is indeed satisfied.

$\underline{\text { Proof of } A_{h}^{(2)}\left(r, r^{\prime}\right)+\mu A_{b}^{(1)}\left(r, r^{\prime}\right)=0}$. Here, we again have $Q_{r} \in \mathbb{R}^{d\left(2 k_{p}-1\right)}$ and $r, r^{\prime} \in \mathbb{Z}^{d\left(2 k_{p}-1\right)}$. We claim that

$$
A_{h}^{(2)}\left(r, r^{\prime}\right)+\mu A_{b}^{(1)}\left(r, r^{\prime}\right)=0
$$

holds.

To this end, we note that

$$
\begin{aligned}
A_{b}^{(1)}\left(r, r^{\prime}\right)= & \frac{1}{2} \operatorname{Tr}_{1}\left(\left(1-\Delta_{x_{1}}\right) \operatorname{Tr}_{2, \ldots, k_{p}} \sum_{j=1}^{k_{p}} B_{j ; k_{p}+1, \ldots, 2 k_{p}-1}^{ \pm} \widetilde{\gamma}_{r, r^{\prime}}^{\left(2 k_{p}-1\right)}\right) \\
= & \frac{1}{2} \sum_{j=2}^{k_{p}} \operatorname{Tr}_{1,2, \ldots, k_{p}}\left(\left(1-\Delta_{x_{1}}\right) B_{j ; k_{p}+1, \ldots, 2 k_{p}-1}^{ \pm} \widetilde{\gamma}_{r, r^{\prime}}^{\left(2 k_{p^{\prime}}-1\right)}\right) \\
& \quad+\frac{1}{2} \operatorname{Tr}_{1,2, \ldots, k_{p}}\left(\left(1-\Delta_{x_{1}}\right) B_{1 ; k_{p}+1, \ldots, 2 k_{p}-1}^{ \pm} \widetilde{\gamma}_{r, r^{\prime}}^{\left(2 k_{p}-1\right)}\right) \\
= & \frac{1}{2} \operatorname{Tr}_{1,2, \ldots, k_{p}}\left(\left(1-\Delta_{x_{1}}\right) B_{1 ; k_{p}+1, \ldots, 2 k_{p}-1}^{ \pm} \widetilde{\gamma}_{r, r^{\prime}}^{\left(2 k_{p}-1\right)}\right) \\
= & \frac{1}{2} \int d x_{1} \ldots d x_{k_{p}} d x_{1}^{\prime} \ldots d x_{k_{p}}^{\prime} \delta\left(x_{1}-x_{1}^{\prime}\right) \cdots \delta\left(x_{k_{p}}-x_{k_{p}}^{\prime}\right) \\
& \left(\left(1-\Delta_{x_{1}}\right) B_{1 ; k_{p}+1, \ldots, 2 k_{p}-1}^{ \pm} \widetilde{\gamma}_{r, r^{\prime}}^{\left(2 k_{p}-1\right)}\right)\left(\underline{x}_{k_{p}}, \underline{x}_{k_{p}}^{\prime}\right) .
\end{aligned}
$$


Using the definition of $B_{1 ; k_{p}+1, \ldots, 2 k_{p}-1}^{ \pm}$and the identity (4.12) from [9], this equals

$$
\begin{aligned}
& A_{b}^{(1)}\left(r, r^{\prime}\right)=\frac{1}{2} \int d x_{1} \ldots d x_{k_{p}} d x_{1}^{\prime} \ldots d x_{k_{p}}^{\prime} \delta\left(x_{1}-x_{1}^{\prime}\right) \cdots \delta\left(x_{k_{p}}-x_{k_{p}}^{\prime}\right) \\
&\left(1+\nabla_{x_{1}} \nabla_{x_{1}^{\prime}}\right) \\
& \widetilde{\gamma}_{r, r^{\prime}}^{\left(2 k_{p}-1\right)}(x_{1}, \ldots, x_{k_{p}}, \underbrace{x_{1}, \ldots, x_{1}}_{k_{p}-1} ; x_{1}^{\prime}, \ldots, x_{k_{p}}^{\prime}, \underbrace{x_{1}, \ldots, x_{1}}_{k_{p}-1}) \\
&\left.\quad \widetilde{\gamma}_{r, r^{\prime}}^{\left(2 k_{p}-1\right)}(x_{1}, \ldots, x_{k_{p}}, \underbrace{x_{1}^{\prime}, \ldots, x_{1}^{\prime}}_{k_{p}-1} ; x_{1}^{\prime}, \ldots, x_{k_{p}}^{\prime}, \underbrace{x_{1}^{\prime}, \ldots, x_{1}^{\prime}}_{k_{p}-1})\right] \\
&=\frac{1}{2} \int d x_{1} \ldots d x_{k_{p}} d x_{1}^{\prime} \ldots d x_{k_{p}}^{\prime} \delta\left(x_{1}-x_{1}^{\prime}\right) \cdots \delta\left(x_{k_{p}}-x_{k_{p}}^{\prime}\right) \\
& \quad\left[\Delta_{x_{1}^{\prime}} \widetilde{\gamma}_{r, r^{\prime}}^{\left(2 k_{p}-1\right)}(x_{1}, \ldots, x_{k_{p}}, \underbrace{x_{1}, \ldots, x_{1}}_{k_{p}-1} ; x_{1}^{\prime}, \ldots, x_{k_{p}}^{\prime}, \underbrace{x_{1}, \ldots, x_{1}}_{k_{p}-1})\right. \\
&\left.\quad-\Delta_{x_{1}} \widetilde{\gamma}_{r, r^{\prime}}^{\left(2 k_{p}-1\right)}(x_{1}, \ldots, x_{k_{p}}, \underbrace{x_{1}^{\prime}, \ldots, x_{1}^{\prime}}_{k_{p}-1} ; x_{1}^{\prime}, \ldots, x_{k_{p}}^{\prime}, \underbrace{x_{1}^{\prime}, \ldots, x_{1}^{\prime}}_{k_{p}-1})\right] .
\end{aligned}
$$

On the other hand, we consider

$$
\begin{aligned}
A_{h}^{(2)}\left(r, r^{\prime}\right)=- & \frac{\mu}{p+2} \operatorname{Tr}_{1}\left(B_{1 ; 2, \ldots, k_{p}}^{+} \sum_{j=1}^{k_{p}}\left(\Delta_{x_{j}}-\Delta_{x_{j}^{\prime}}\right) \operatorname{Tr}_{k_{p}+1 \ldots 2 k_{p}-1} \widetilde{\gamma}_{r, r^{\prime}}^{\left(2 k_{p}-1\right)}\right) \\
=- & \frac{\mu}{p+2} \int d x_{1} \ldots d x_{k_{p}} d x_{1}^{\prime} \ldots d x_{k_{p}}^{\prime} \delta\left(x_{1}=\ldots=x_{k_{p}}=x_{1}^{\prime}=\ldots=x_{k_{p}}^{\prime}\right) \\
& \sum_{j=1}^{k_{p}}\left(\Delta_{x_{j}}-\Delta_{x_{j}^{\prime}}\right) \operatorname{Tr}_{k_{p}+1 \ldots 2 k_{p}-1} \widetilde{\gamma}_{r, r^{\prime}}^{\left(2 k_{p}-1\right)}\left(\underline{x}_{2 k_{p}-1} ; \underline{x}_{2 k_{p}-1}^{\prime}\right)
\end{aligned}
$$

By symmetry of $\widetilde{\gamma}^{\left(k_{p}\right)}$ with respect to the components of $\underline{x}_{k_{p}}$ and $\underline{x}_{k_{p}}^{\prime}$, this yields

$$
\begin{aligned}
A_{h}^{(2)}\left(r, r^{\prime}\right)=- & \frac{\mu k_{p}}{p+2} \int d x_{1} \ldots d x_{k_{p}} d x_{1}^{\prime} \ldots d x_{k_{p}}^{\prime} \delta\left(x_{1}=\ldots=x_{k_{p}}=x_{1}^{\prime}=\ldots=x_{k_{p}}^{\prime}\right) \\
& \left(\Delta_{x_{1}}-\Delta_{x_{1}^{\prime}}\right) \operatorname{Tr}_{k_{p} \ldots 2 k_{p}-1} \widetilde{\gamma}_{r, r^{\prime}}^{\left(2 k_{p^{\prime}}-1\right)}\left(\underline{x}_{2 k_{p}-1} ; \underline{x}_{2 k_{p}-1}^{\prime}\right) \\
=- & \frac{\mu}{2} \int d x_{1} \ldots d x_{k_{p}} d x_{1}^{\prime} \ldots d x_{k_{p}}^{\prime} \delta\left(x_{1}-x_{1}^{\prime}\right) \ldots \delta\left(x_{k_{p}}-x_{k_{p}}^{\prime}\right) \\
& {\left[\Delta_{x_{1}^{\prime}} \widetilde{\gamma}_{r, r^{\prime}}^{\left(2 k_{p}\right)}(x_{1}, \ldots, x_{k_{p}}, \underbrace{x_{1}, \ldots, x_{1}}_{k_{p}-1} ; x_{1}^{\prime}, \ldots, x_{k_{p}}^{\prime}, \underbrace{x_{1}, \ldots, x_{1}}_{k_{p}-1})\right.} \\
& \left.-\Delta_{x_{1}} \widetilde{\gamma}_{r, r^{\prime}}^{\left(2 k_{p}\right)}(x_{1}, \ldots, x_{k_{p}}, \underbrace{x_{1}^{\prime}, \ldots, x_{1}^{\prime}}_{k_{p}-1} ; x_{1}^{\prime}, \ldots, x_{k_{p}}^{\prime}, \underbrace{x_{1}^{\prime}, \ldots, x_{1}^{\prime}}_{k_{p}-1})\right]
\end{aligned}
$$

where we exchanged the roles of $\left(x_{j}, x_{j}^{\prime}\right)$ for $j=1, \ldots, k_{p}$ with those of $\left(x_{j}, x_{j}^{\prime}\right)$ for $j=k_{p}+1, \ldots, 2 k_{p}$. 
Similarly as above, one can prove that each term in the difference is separately finite, that is,

$$
A_{h}^{(2)}\left(r, r^{\prime}\right)=A_{h,+}^{(2)}\left(r, r^{\prime}\right)-A_{h,-}^{(2)}\left(r, r^{\prime}\right)
$$

where both

$$
\begin{gathered}
A_{h,+}^{(2)}\left(r, r^{\prime}\right) \\
:=\frac{\mu k_{p}}{p+2} \int_{Q_{r} \times Q_{r^{\prime}}} d u_{1} \ldots d u_{2 k_{p}-1} d u_{1}^{\prime} \ldots d u_{2 k_{p}-1}^{\prime} \delta\left(u_{2}-u_{2}^{\prime}\right) \cdots \delta\left(u_{k_{p}}-u_{k_{p}}^{\prime}\right) u_{1}^{2} \\
\delta\left(u_{1}-u_{1}^{\prime}+\sum_{j=k_{p}+1}^{2 k_{p}-1}\left(u_{j}-u_{j}^{\prime}\right)\right) \widetilde{\gamma}^{\left(2 k_{p}-1\right)}\left(\underline{u}_{2 k_{p}-1} ; \underline{u}_{2 k_{p}-1}^{\prime}\right)
\end{gathered}
$$

and

$$
\begin{aligned}
& A_{h,-}^{(2)}\left(r, r^{\prime}\right) \\
& :=\frac{\mu k_{p}}{p+2} \int_{Q_{r} \times Q_{r^{\prime}}} d u_{1} \ldots d u_{2 k_{p}-1} d u_{1}^{\prime} \ldots d u_{2 k_{p}-1}^{\prime} \delta\left(u_{2}-u_{2}^{\prime}\right) \cdots \delta\left(u_{k_{p}}-u_{k_{p}}^{\prime}\right)\left(u_{1}^{\prime}\right)^{2} \\
& \quad \delta\left(u_{1}-u_{1}^{\prime}+\sum_{j=k_{p}+1}^{2 k_{p}-1}\left(u_{j}-u_{j}^{\prime}\right)\right) \widehat{\left.\widetilde{\gamma}^{\left(2 k_{p}-1\right.}\right)}\left(\underline{u}_{2 k_{p}-1} ; \underline{u}_{2 k_{p}-1}^{\prime}\right)
\end{aligned}
$$

are bounded for any $\left(r, r^{\prime}\right)$. The proof is straightforwardly obtained from adapting the proof of $A_{b}^{(2)}\left(r, r^{\prime}\right)=0$, by substituting $\widetilde{\gamma}^{\left(2 k_{p}-1\right)} \rightarrow u_{1}^{2} \widetilde{\gamma}^{\left(2 k_{p}-1\right)}$, respectively $\widetilde{\gamma}^{\left(2 k_{p}-1\right)} \rightarrow\left(u_{1}^{\prime}\right)^{2} \widetilde{\gamma}^{\left(2 k_{p}-1\right)}$ in the arguments given there. The result is that

$$
A_{h, \pm}^{(2)}\left(r, r^{\prime}\right)<C\left(\operatorname{Tr}\left(\widetilde{\gamma}_{r, r}^{\left(2 k_{p}-1\right)}\right)+\operatorname{Tr}\left(\widetilde{\gamma}_{r^{\prime}, r^{\prime}}^{\left(2 k_{p}-1\right)}\right)\right)<C^{\prime} .
$$

We shall not repeat the details here.

As a consequence, we obtain that

$$
\mu A_{b}^{(1)}\left(r, r^{\prime}\right)+A_{h}^{(2)}\left(r, r^{\prime}\right)=0,
$$

by comparing terms in (4.42) and (4.45). This proves (4.38).

Collecting the above results, we arrive at

$$
\begin{aligned}
& A(1 ; m) \\
& \quad=\sum_{r, r^{\prime} \in \mathbb{Z}^{d k_{p}}} A_{h}^{(1)}\left(r, r^{\prime}\right)+\sum_{r, r^{\prime} \in \mathbb{Z}^{d\left(2 k_{p}-1\right)}}\left(\mu A_{b}^{(1)}\left(r, r^{\prime}\right)+A_{h}^{(2)}\left(r, r^{\prime}\right)+A_{b}^{(2)}\left(r, r^{\prime}\right)\right) \\
& \quad=0
\end{aligned}
$$

and similarly, $A(\ell ; m)=0$ for all $\ell=2, \ldots, m$ (see (4.11)). Therefore,

$$
\partial_{t}\left\langle\mathcal{K}^{(m)}\right\rangle_{\Gamma(t)}=\sum_{\ell=1}^{m}[A(\ell ; m)]=0 .
$$

Consequently, we have proved (4.6). 


\section{Generalized Sobolev and Gagliardo-Nirenberg inequalities}

As a preparation for Section 6 where we use higher order energy conservation to obtain a priori bounds on the $\mathfrak{H}_{\xi}^{1}$-norm of solutions of $p$-GP hierarchies, we present a generalization of Sobolev and Gagliardo-Nirenberg inequalities on the level of density matrices. We remark that W. Beckner recently derived a family of sharp estimates in [5] related to results of this type.

Theorem 5.1. (Sobolev inequality) Assume that $f \in H^{\alpha}\left(\mathbb{R}^{q d}\right)$ for $\alpha>\alpha_{0}:=$ $\frac{(q-1) d}{2 q}<1$. Then, there exists a constant $C(d, q, \alpha)$ such that

$$
\begin{aligned}
& \left(\int d x|f(\underbrace{x, \ldots, x}_{q})|^{2}\right)^{\frac{1}{2}} \\
& \leq C(d, q, \alpha)\left(\int d x_{1} \cdots d x_{q}\left|\left\langle\nabla_{x_{1}}\right\rangle^{\alpha} \cdots\left\langle\nabla_{x_{q}}\right\rangle^{\alpha} f\left(x_{1}, \ldots, x_{q}\right)\right|^{2}\right)^{\frac{1}{2}} \\
& =\|f\|_{H_{x_{1}, \ldots, x_{q}}^{\alpha}}
\end{aligned}
$$

for $x_{i} \in \mathbb{R}^{d}$. In particular, $C(d, q, \alpha)<C^{\prime}(d, q)\left|\alpha-\alpha_{0}\right|^{-q / 2}$.

Proof. We use a Littlewood-Paley decomposition $1=\sum_{j} P_{j}$ where $P_{j}$ acts in frequency space as multiplication with the characteristic function on the dyadic annulus $A_{i}:=\left\{\xi \in \mathbb{R}^{d}\left|2^{j} \leq\right| \xi \mid<2^{j+1}\right\}$, and $P_{0}$ is the characteristic function on the unit ball.

Let $j_{1}, \ldots, j_{q} \in \mathbb{N}_{0}$, and

$$
f_{j_{1} \ldots j_{q}}\left(x_{1}, \ldots, x_{q}\right):=\left(P_{j_{1}}^{(1)} \cdots P_{j_{q}}^{(q)} f\right)\left(x_{1}, \ldots, x_{q}\right)
$$

where the superscript in $P_{i}^{(m)}$ signifies that it acts on the $m$-th variable.

Then, clearly, the Fourier transform satisfies

$$
\widehat{f_{j_{1} \ldots j_{q}}}\left(\xi_{1}, \ldots, \xi_{q}\right)=h_{j_{1}}\left(\xi_{1}\right) \cdots h_{j_{q}}\left(\xi_{q}\right) \widehat{f_{j_{1} \ldots j_{q}}}\left(\xi_{1}, \ldots, \xi_{q}\right)
$$

where $h_{i}$ are Schwartz class functions with $P_{i} h_{i}=P_{i}$.

We note that

$$
\begin{aligned}
h_{j}^{\vee}(x) & =\int d \xi h_{j}(\xi) e^{2 \pi i \xi x} \\
& =2^{j d} \int d \xi h_{1}(\xi) e^{2 \pi i \xi\left(2^{j} x\right)} \\
& =2^{j d} h_{1}^{\vee}\left(2^{j} x\right) .
\end{aligned}
$$

Therefore, $h_{j}^{\vee}$ is a smooth delta function with amplitude $2^{j d}$, and supported on a ball of radius $2^{-j}$. In particular,

$$
\left\|h_{j}^{\vee}\right\|_{L_{x}^{\infty}} \leq c 2^{j d},\left\|h_{j}^{\vee}\right\|_{L_{x}^{1}}=\left\|h_{1}^{\vee}\right\|_{L_{x}^{1}} \leq c^{\prime},
$$

for constants $c, c^{\prime}$ independent of $j$. Because $h_{j}$ is an even function for all $j$, it follows that $h_{j}^{\vee} \in \mathbb{R}$. 
Accordingly, performing the inverse Fourier transform,

$$
\begin{aligned}
f_{j_{1} \ldots j_{q}}\left(x_{1}, \ldots, x_{q}\right)=\int d y_{1} \cdots & d y_{q} f_{j_{1} \ldots j_{q}}\left(y_{1}, \ldots, y_{q}\right) \\
& h_{j_{1}}^{\vee}\left(y_{1}+x_{1}\right) \cdots h_{j_{q}}^{\vee}\left(y_{q}+x_{q}\right) .
\end{aligned}
$$

In particular,

$$
\begin{gathered}
\int d x\left|f_{j_{1} \ldots j_{q}}(x, \ldots, x)\right|^{2} \\
=\int d y_{1} \cdots d y_{q} d y_{1}^{\prime} \cdots d y_{q}^{\prime} f_{j_{1} \ldots j_{q}}\left(y_{1}, \ldots, y_{q}\right) \overline{f_{j_{1} \ldots j_{q}}\left(y_{1}^{\prime}, \ldots, y_{q}^{\prime}\right)} \\
\int d x h_{j_{1}}^{\vee}\left(y_{1}+x\right) \cdots h_{j_{q}}^{\vee}\left(y_{q}+x\right) \\
h_{j_{1}}^{\vee}\left(y_{1}^{\prime}+x\right) \cdots h_{j_{q}}^{\vee}\left(y_{q}^{\prime}+x\right) .
\end{gathered}
$$

Using Cauchy-Schwarz only on $f_{j_{1} \ldots j_{q}} \overline{f_{j_{1} \ldots j_{q}}}$, this is bounded by

$$
\begin{aligned}
\int d x\left|f_{j_{1} \ldots j_{q}}(x, \ldots, x)\right|^{2} \leq & \int d y_{1} \cdots d y_{q}\left|f_{j_{1} \ldots j_{q}}\left(y_{1}, \ldots, y_{q}\right)\right|^{2} \\
& \int d x \int d y_{1}^{\prime} \cdots d y_{q}^{\prime} \mid h_{j_{1}}^{\vee}\left(y_{1}+x\right) \cdots h_{j_{q}}^{\vee}\left(y_{q}+x\right) \\
& h_{j_{1}}^{\vee}\left(y_{1}^{\prime}+x\right) \cdots h_{j_{q}}^{\vee}\left(y_{q}^{\prime}+x\right) \mid .
\end{aligned}
$$

Thus, integrating out $y_{1}^{\prime}, \ldots, y_{q}^{\prime}$ and using $\left\|h_{j}^{\vee}\right\|_{L_{x}^{1}}<c^{\prime}$,

$$
\begin{aligned}
\int d x\left|f_{j_{1} \ldots j_{q}}(x, \ldots, x)\right|^{2} \leq & C \int d y_{1} \cdots d y_{q}\left|f_{j_{1} \ldots j_{q}}\left(y_{1}, \ldots, y_{q}\right)\right|^{2} \\
& \int d x\left|h_{j_{1}}^{\vee}\left(y_{1}+x\right) \cdots h_{j_{q}}^{\vee}\left(y_{q}+x\right)\right| .
\end{aligned}
$$

Now, we assume without any loss of generality that $j_{i} \leq j_{q}$ for all $i<q$. Then,

$$
\begin{aligned}
\int d x\left|h_{j_{1}}^{\vee}\left(y_{1}+x\right) \cdots h_{j_{q}}^{\vee}\left(y_{q}+x\right)\right| & \leq\left\|h_{j_{1}}^{\vee}\right\|_{L_{x}^{\infty}} \cdots\left\|h_{j_{q-1}}^{\vee}\right\|_{L_{x}^{\infty}}\left\|h_{j_{q}}^{\vee}\right\|_{L_{x}^{1}} \\
& \leq 2^{\left(j_{1}+\cdots+j_{q-1}\right) d} c^{\prime} .
\end{aligned}
$$

Now, since by assumption, $j_{i} \leq j_{q}$ for all $i<q$,

$$
j_{1}+\cdots+j_{q-1} \leq \frac{q-1}{q}\left(j_{1}+\cdots+j_{q}\right) .
$$

Therefore,

$$
\begin{aligned}
& \int d x\left|f_{j_{1} \ldots j_{q}}(x, \ldots, x)\right|^{2} \\
& \quad \leq C 2^{2\left(j_{1}+\cdots+j_{q}\right) \alpha_{0}} \int d y_{1} \cdots d y_{q}\left|f_{j_{1} \ldots j_{q}}\left(y_{1}, \ldots, y_{q}\right)\right|^{2},
\end{aligned}
$$

where

$$
\alpha_{0}:=\frac{(q-1) d}{2 q}
$$


We thus find that

$$
\begin{aligned}
\| & f(x, \ldots, x) \|_{L_{x}^{2}\left(\mathbb{R}^{d}\right)} \\
& =\left\|\sum_{j_{1}, \ldots, j_{q}} f_{j_{1} \ldots j_{q}}(x, \ldots, x)\right\|_{L_{x}^{2}\left(\mathbb{R}^{d}\right)} \\
& \leq \sum_{j_{1}, \ldots, j_{q}}\left(\int d x\left|f_{j_{1}, \ldots, j_{q}}(x, \ldots, x)\right|^{2}\right)^{\frac{1}{2}} \\
& \leq C \sum_{j_{1}, \ldots, j_{q}} 2^{\left(j_{1}+\cdots+j_{q}\right) \alpha_{0}}\left(\int d y_{1} \cdots d y_{q}\left|f_{j_{1}, \ldots, j_{q}}\left(y_{1}, \ldots, y_{q}\right)\right|^{2}\right)^{\frac{1}{2}} \\
& =C\left(\sum_{j_{1}, \ldots, j_{q}} 2^{-2\left(j_{1}+\cdots+j_{q}\right) \epsilon}\right)^{\frac{1}{2}}\left(\sum_{j_{1}, \ldots, j_{q}} 2^{2\left(j_{1}+\cdots+j_{q}\right) \alpha}\left\|f_{j_{1}, \ldots, j_{q}}\right\|_{L_{x_{1}, \ldots, x_{q}}^{2}}^{2}\right)^{\frac{1}{2}} \\
& =C_{\epsilon}\|f\|_{H_{x_{1}, \ldots, x_{q}}^{\alpha}} .
\end{aligned}
$$

for $\alpha=\alpha_{0}+\epsilon$, and any $\epsilon>0$. We note that from the above, $C_{\epsilon} \leq C \epsilon^{-q}$ follows immediately. This is the asserted result.

We immediately obtain the following Gagliardo-Nirenberg type inequality.

Theorem 5.2. (Gagliardo-Nirenberg inequality) Assume that $f \in H^{1}\left(\mathbb{R}^{q d}\right)$ and $\alpha>\alpha_{0}:=\frac{(q-1) d}{2 q}<1$. Then, there exists a constant $C(d, q, \alpha)$ such that

$$
\left(\int d x|f(\underbrace{x, \ldots, x}_{q})|^{2}\right)^{\frac{1}{2}} \leq C(d, q, \alpha)\|f\|_{H_{x_{1}, \ldots, x_{q}}^{1}}^{\alpha}\|f\|_{L_{x_{1}, \ldots, x_{q}}^{2}}^{1-\alpha}
$$

where $x_{i} \in \mathbb{R}^{d}$. In particular, $C(d, q, \alpha)<C^{\prime}(d, q)\left|\alpha-\alpha_{0}\right|^{-q / 2}$.

Proof. From (5.14), the Hölder estimate yields

$$
\begin{aligned}
& \|f(x, \ldots, x)\|_{L_{x}^{2}\left(\mathbb{R}^{d}\right)} \\
& \leq \sum_{j_{1}, \ldots, j_{q}}\left\|f_{j_{1} \ldots j_{q}}(x, \ldots, x)\right\|_{L_{x}^{2}\left(\mathbb{R}^{d}\right)} \\
& \leq C \sum_{j_{1}, \ldots, j_{q}} 2^{\left(j_{1}+\cdots+j_{q}\right) \alpha_{0}}\left\|f_{j_{1}, \ldots, j_{q}}\right\|_{L^{2}} \\
& =C \sum_{j_{1}, \ldots, j_{q}} 2^{\left(j_{1}+\cdots+j_{q}\right) \alpha_{0}}\left\|f_{j_{1}, \ldots, j_{q}}\right\|_{L^{2}}^{\alpha}\left\|f_{j_{1}, \ldots, j_{q}}\right\|_{L^{2}}^{1-\alpha} \\
& \leq C\left[\sum_{j_{1}, \ldots, j_{q}}\left(2^{\left(j_{1}+\cdots+j_{q}\right)\left(\alpha_{0}+\delta\right)}\left\|f_{j_{1}, \ldots, j_{q}}\right\|_{L^{2}}^{\alpha}\right)^{\frac{1}{\alpha}}\right]^{\alpha} \\
& \quad\left[\sum_{j_{1}, \ldots, j_{q}}\left(2^{-\left(j_{1}+\cdots+j_{q}\right) \delta}\left\|f_{j_{1}, \ldots, j_{q}}\right\|_{L^{2}}^{1-\alpha}\right)^{\frac{1}{1-\alpha}}\right]^{1-\alpha} .
\end{aligned}
$$


Letting $\epsilon=\delta>0$ and $1>\alpha \geq \alpha_{0}+2 \epsilon$, where $\alpha_{0}=\frac{(q-1) d}{2 q}<1$, this is bounded by

$$
\begin{gathered}
\leq C\left[\left(\sum_{j_{1}, \ldots, j_{q}} 2^{-2\left(j_{1}+\cdots+j_{q}\right) \frac{\epsilon}{\alpha}}\right)^{\frac{1}{2}}\left(\sum_{j_{1}, \ldots, j_{q}} 2^{2\left(j_{1}+\cdots+j_{q}\right) \frac{\alpha_{0}+\delta+\epsilon}{\alpha}}\left\|f_{j_{1}, \ldots, j_{q}}\right\|_{L^{2}}^{2}\right)^{\frac{1}{2}}\right]^{\alpha} \\
\quad\left[\left(\sum_{j_{1}, \ldots, j_{q}} 2^{-2\left(j_{1}+\cdots+j_{q}\right) \frac{\delta}{1-\alpha}}\right)^{\frac{1}{2}}\left(\sum_{j_{1}, \ldots, j_{q}}\left\|f_{j_{1}, \ldots, j_{q}}\right\|_{L^{2}}^{2}\right)^{\frac{1}{2}}\right]^{1-\alpha} \\
\leq C\left[\left(\frac{\epsilon}{\alpha}\right)^{-\frac{q}{2}}\left(\sum_{j_{1}, \ldots, j_{q}}\left\|f_{j_{1}, \ldots, j_{q}}\right\|_{H}^{2}{ }_{H_{0}+\delta+\epsilon}^{\frac{\alpha_{0}}{\alpha}}\right)^{\frac{1}{2}}\right]^{\alpha} \\
\quad\left[\left(\frac{\delta}{1-\alpha}\right)^{-\frac{q}{2}}\left(\sum_{j_{1}, \ldots, j_{q}}\left\|f_{j_{1}, \ldots, j_{q}}\right\|_{L^{2}}^{2}\right)^{\frac{1}{2}}\right]^{1-\alpha} \\
\leq C\left(\alpha^{\alpha}(1-\alpha)^{1-\alpha} \frac{1}{\epsilon}\right)^{q / 2}\|f\|_{H_{x_{1}, \ldots, x_{q}}^{1}}^{\alpha}\|f\|_{L_{x_{1}, \ldots, x_{q}}^{2}}^{1-\alpha} \\
\leq C \epsilon^{-q / 2}\|f\|_{H_{x_{1}, \ldots, x_{q}}^{1}}^{\alpha}\|f\|_{L_{x_{1}, \ldots, x_{q}}^{2}}^{1-\alpha}
\end{gathered}
$$

Here we observed that $\frac{1}{\alpha}, \frac{1}{1-\alpha}>1$ are Hölder conjugate exponents, and we used that $\sup _{\tau \in[0,1]} \tau^{\tau}=1$.

We note that $\alpha_{0}=\frac{d}{4}$ for the cubic, $p=2$, and $\alpha_{0}=\frac{3 d}{8}$ for the quintic case, $p=4$. Accordingly, one may choose $\epsilon \geq \frac{1}{5}$ in both cases (i.e., $\epsilon$ is not very small), where $d \leq 3$ for the cubic, and $d \leq 2$ for the quintic case.

\section{A PRIORI ENERGY BOUNDS}

In this section we use higher order energy functionals to obtain three types of bounds:

(1) A priori energy bounds for both focusing and defocusing energy-subcritical p-GP hierarchies (Subsection 6.1).

(2) A priori $H^{1}$ bounds for defocusing energy subcritical p-GP hierarchies (Subsection 6.2).

(3) A priori $H^{1}$ bounds for $L^{2}$-subcritical focusing p-GP hierarchies (Subsection 6.3).

First, we present a priori energy bounds which are valid for solutions of the focusing and defocusing energy-subcritical $p$-GP hierarchies.

\subsection{A priori energy bounds for focusing and defocusing energy-subcritical p-GP hierarchies.}

Theorem 6.1. Let $p<\frac{4}{d-2}$. If $\Gamma(t) \in \mathfrak{H}_{\xi^{\prime}}^{1}$ is a solution to the $p$-GP hierarchy (focusing or defocusing), then the a priori bound

$$
\sum_{m \in \mathbb{N}}(2 \xi)^{m}\left\langle\mathcal{K}^{(m)}\right\rangle_{\Gamma(t)} \leq\|\Gamma(t)\|_{\mathfrak{H}_{\xi^{\prime}}^{1}}
$$


holds for all $\xi$ satisfying

$$
\xi \leq\left(1+\frac{2}{p+2} C_{S o b}(d, p)\right)^{-\frac{1}{k_{p}}} \xi^{\prime}
$$

and all $t \in \mathbb{R}$.

Proof. Let $p<\frac{4}{d-2}$. We shall use the Sobolev inequalities for the GP hierarchy, Theorem 5.1 to bound the interaction energy by the kinetic energy:

$$
\operatorname{Tr}_{1}\left(B_{1 ; 2, \ldots, k_{p}} \widetilde{\gamma}^{\left(k_{p}\right)}\right) \leq C_{S o b}(d, p) \operatorname{Tr}_{1, \ldots, k_{p}}\left(S^{\left(k_{p}, 1\right)} \widetilde{\gamma}^{\left(k_{p}\right)}\right) .
$$

To see this, we write $\widetilde{\gamma}^{\left(k_{p}\right)}$ as

$$
\widetilde{\gamma}^{\left(k_{p}\right)}\left(\underline{x}_{k_{p}}, \underline{x}_{k_{p}}^{\prime}\right)=\sum_{j} \lambda_{j}\left|\phi_{j}\left(\underline{x}_{k_{p}}\right)\right\rangle\left\langle\phi_{j}\left(\underline{x}_{k_{p}}^{\prime}\right)\right|,
$$

with respect to an orthonormal basis $\left(\phi_{j}\right)_{j}$, where $\lambda_{j} \geq 0$ and $\sum_{j} \lambda_{j}=1$. Then we have

$$
\operatorname{Tr}_{1}\left(B_{1 ; 2, \ldots, k_{p}}^{+} \widetilde{\gamma}^{\left(k_{p}\right)}\right)=\sum_{j} \lambda_{j} \int d x\left|\phi_{j}(\underbrace{x, \ldots, x}_{k_{p}})\right|^{2} .
$$

Now Theorem 5.1 (with $q=k_{p}$ and $\alpha=1$ ) implies that for $p<\frac{4}{d-2}$ we have

$$
\begin{aligned}
\operatorname{Tr}_{1}\left(B_{1 ; 2, \ldots, k_{p}}^{+} \widetilde{\gamma}^{\left(k_{p}\right)}\right) \\
\quad \leq C_{S o b} \sum_{j} \lambda_{j}\left\|\phi_{j}\right\|_{\underline{\underline{x}}_{\underline{k}_{p}}^{1}}^{2} \\
\leq C_{S o b} \sum_{j} \lambda_{j}\left\|\phi_{j}\right\|_{\mathfrak{h}_{\underline{x}_{k_{p}}}^{1}}^{2} \\
\quad=C_{S o b} \operatorname{Tr}_{1, \ldots, k_{p}}\left(S^{\left(k_{p}, 1\right)} \widetilde{\gamma}^{\left(k_{p}\right)}\right) .
\end{aligned}
$$

Accordingly, (6.3) implies

$$
\operatorname{Tr}_{1}\left(K_{1} \widetilde{\gamma}^{\left(k_{p}\right)}\right) \leq\left(\frac{1}{2}+\frac{1}{p+2} C_{S o b}(d, p)\right) \operatorname{Tr}_{1, \ldots, k_{p}}\left(S^{\left(k_{p}, 1\right)} \widetilde{\gamma}^{\left(k_{p}\right)}\right) .
$$

By iteration, we obtain that

$$
\left\langle\mathcal{K}^{(m)}\right\rangle_{\Gamma} \leq\left(\frac{1}{2}+\frac{1}{p+2} C_{S o b}(d, p)\right)^{m} \operatorname{Tr}_{1, \ldots, m k_{p}}\left(S^{\left(m k_{p}, 1\right)} \gamma^{\left(m k_{p}\right)}\right) .
$$

Therefore,

$$
\begin{aligned}
\sum_{\ell}(2 \xi)^{\ell}\left\langle\mathcal{K}^{(\ell)}\right\rangle_{\Gamma} & \leq \sum_{\ell}\left(\left(1+\frac{2}{p+2} C_{S o b}(d, p)\right)^{\frac{1}{k_{p}}} \xi\right)^{\ell}\left\|\gamma^{(\ell)}\right\|_{\mathfrak{h}_{\ell}^{1}} \\
& \leq\|\Gamma\|_{\mathfrak{H}_{\xi^{\prime}}^{1}}
\end{aligned}
$$

for all $\xi$ satisfying

$$
\xi \leq\left(1+\frac{2}{p+2} C_{S o b}(d, p)\right)^{-\frac{1}{k_{p}}} \xi^{\prime} .
$$

Hence, the claim follows. 
6.2. A priori $H^{1}$ bounds for defocusing energy subcritical GP hierarchies. For energy subcritical, defocusing GP hierarchies, we can now deduce a priori energy bounds as follows.

Theorem 6.2. Assume that $\mu=+1$ (defocusing), $p<\frac{4}{d-2}$, and that $\Gamma(t) \in \mathfrak{H}_{\xi}^{1}$, $t \in[0, T]$, is a positive semidefinite solution of the $p$-GP hierarchy with initial data $\Gamma_{0} \in \mathfrak{H}_{\xi^{\prime}}^{1}$ for

$$
\xi \leq\left(1+\frac{2}{p+2} C_{S o b}(d, p)\right)^{-\frac{1}{k_{p}}} \xi^{\prime}
$$

Then, one finds

$$
\begin{aligned}
\|\Gamma(t)\|_{\mathfrak{H}_{\xi}^{1}} & \leq \sum_{m \in \mathbb{N}}(2 \xi)^{m}\left\langle\mathcal{K}^{(m)}\right\rangle_{\Gamma(t)} \\
& =\sum_{m \in \mathbb{N}}(2 \xi)^{m}\left\langle\mathcal{K}^{(m)}\right\rangle_{\Gamma_{0}} \leq\left\|\Gamma_{0}\right\|_{\mathfrak{H}_{\xi^{\prime}}^{1}}
\end{aligned}
$$

for all $t \in[0, T]$.

Proof. We first note that for the defocusing $p$-GP hierarchy, it follows immediately from Theorem 4.1 that

$$
\begin{aligned}
\|\Gamma(t)\|_{\mathfrak{H}_{\xi}^{1}} & \leq \sum_{m \in \mathbb{N}}(2 \xi)^{m}\left\langle\mathcal{K}^{(m)}\right\rangle_{\Gamma(t)} \\
& =\sum_{m \in \mathbb{N}}(2 \xi)^{m}\left\langle\mathcal{K}^{(m)}\right\rangle_{\Gamma_{0}}
\end{aligned}
$$

for $t \in[0, T]$. The first inequality is obtained by discarding all of the (positive) interaction energies in $\left\langle\mathcal{K}^{(m)}\right\rangle_{\Gamma(t)}$.

Subsequently, we use the a priori bound (6.1) derived in Theorem 6.1 to obtain (6.12).

6.3. A priori energy bounds for $L^{2}$ subcritical focusing GP hierarchies. In this subsection, we prove a priori $\mathfrak{H}_{\xi}^{1}$-bounds for focusing $L^{2}$ subcritical $p$-GP hierarchies, $p<p_{L^{2}}=\frac{4}{d}$. The defocusing case is already contained in Theorem 6.2. The analogous result for the NLS is well-known, and is based on the use of energy conservation where the $H^{1}$ norm (if large) of the solution is seen to dominate over the potential energy, via the Gagliardo-Nirenberg inequality. We obtain the following similar result in the context of the $L^{2}$-subcritical $p$-GP hierarchy.

Theorem 6.3. Let $p<p_{L^{2}}=\frac{4}{d}\left(L^{2}\right.$ subcritical). Moreover, let $\alpha>\alpha_{0}:=\frac{\left(k_{p}-1\right) d}{2 k_{p}}$ and $\alpha k_{p}<1$, where $k_{p}=1+\frac{p}{2}$, and $\alpha<1$. Let

$$
D:=D(\alpha, p, d,|\mu|)=\left(1-|\mu| \frac{C_{0}(\alpha)}{1-4^{-\left(1-\alpha k_{p}\right)}}\right),
$$

where $C_{0}(\alpha)$ is characterized in (6.20).

Assume that $\Gamma(t) \in \mathfrak{H}_{\xi}^{1}$ is a positive semidefinite solution of the focusing $(\mu<0)$ $p$-GP hierarchy for $t \in[0, T]$, given initial data $\Gamma(0)=\Gamma_{0} \in \mathfrak{H}_{\xi^{\prime}}^{1}$ where

$$
\xi \leq \frac{1}{D}\left(1+\frac{2}{p+2} C_{S o b}(d, p)\right)^{-\frac{1}{k_{p}}} \xi^{\prime} .
$$


If $\mu<0$ is such that

$$
|\mu|<\frac{1-4^{-\left(1-\alpha k_{p}\right)}}{C_{0}(\alpha)}
$$

then the a priori bound

$$
\begin{aligned}
\|\Gamma(t)\|_{\mathfrak{H}_{\xi}^{1}} & \leq \sum_{m=1}^{\infty}(2 D \xi)^{m}\left\langle\mathcal{K}^{(m)}\right\rangle_{\Gamma(t)} \\
& =\sum_{m=1}^{\infty}(2 D \xi)^{m}\left\langle\mathcal{K}^{(m)}\right\rangle_{\Gamma_{0}} \\
& \leq\left\|\Gamma_{0}\right\|_{\mathfrak{H}_{\xi^{\prime}}^{1}}
\end{aligned}
$$

holds for all $t \in[0, T]$.

Proof. First, we observe that it follows immediately from (6.1) that (6.18) implies (6.19).

Next, we show that $\|\Gamma(t)\|_{\mathfrak{H}_{\xi}^{1}}$ is bounded by the right hand side in (6.17). Given $\alpha>\alpha_{0}=\frac{\left(k_{p}-1\right) d}{2 k_{p}}$ where $\alpha_{0}<1$, we infer from Theorem 5.1 that

$$
\operatorname{Tr}_{1}\left(B_{1 ; 2, \ldots, k_{p}}^{+} \gamma^{\left(k_{p}\right)}\right) \leq C_{0}(\alpha) \operatorname{Tr}\left(S^{\left(k_{p}, \alpha\right)} \gamma^{\left(k_{p}\right)}\right),
$$

as follows. We write $\gamma^{\left(k_{p}\right)}$ with respect to an orthonormal eigenbasis $\left(\phi_{j}\right)_{j}$,

$$
\gamma^{\left(k_{p}\right)}\left(\underline{x}_{k_{p}}, \underline{x}_{k_{p}}^{\prime}\right)=\sum_{j} \lambda_{j}\left|\phi_{j}\left(\underline{x}_{k_{p}}\right)\right\rangle\left\langle\phi_{j}\left(\underline{x}_{k_{p}}^{\prime}\right)\right|,
$$

where $\lambda_{j} \geq 0$ and $\sum_{j} \lambda_{j}=1$, so that

$$
\operatorname{Tr}_{1}\left(B_{1 ; 2, \ldots, k_{p}}^{+} \gamma^{\left(k_{p}\right)}\right)=\sum_{j} \lambda_{j} \int d x\left|\phi_{j}(\underbrace{x, \ldots, x}_{k_{p}})\right|^{2} .
$$

Then Theorem [5.1 (with $q=k_{p}$ ) implies that

$$
\begin{aligned}
& \operatorname{Tr}_{1}\left(B_{1 ; 2, \ldots, k_{p}}^{+} \gamma^{\left(k_{p}\right)}\right) \\
& \leq C_{0}(\alpha) \sum_{j} \lambda_{j}\left\|\phi_{j}\right\|_{\underline{\mathfrak{h}}_{\underline{x}_{k_{p}}}^{\alpha}}^{2} \\
& \leq C_{0}(\alpha) \operatorname{Tr}\left(S^{\left(k_{p}, \alpha\right)} \gamma^{\left(k_{p}\right)}\right),
\end{aligned}
$$

which is what we claimed in (6.20).

Next, we recall the definition of the operators

$$
K_{\ell}=K_{\ell}^{(1)}+K_{\ell}^{(2)}
$$

where

$$
K_{\ell}^{(1)}:=\frac{1}{2}\left(1-\Delta_{x_{\ell}}\right) \operatorname{Tr}_{\ell+1, \ldots, \ell+\frac{p}{2}}
$$

and

$$
K_{\ell}^{(2)}:=\frac{\mu}{p+2} B_{\ell ; \ell+1, \ldots, \ell+\frac{p}{2}}^{+}
$$


for $\ell \in \mathbb{N}$. Moreover, we have

$$
\mathcal{K}^{(m)}:=K_{1} K_{k_{p}+1} \cdots K_{(m-1) k_{p}+1}
$$

and we proved that

$$
\left\langle\mathcal{K}^{(m)}\right\rangle_{\Gamma(t)}:=\operatorname{Tr}_{1, k_{p}+1,2 k_{p}+1, \ldots,(m-1) k_{p}+1}\left(\mathcal{K}^{(m)} \gamma^{\left(m k_{p}\right)}(t)\right)
$$

is a conserved quantity, for every $m \in \mathbb{N}$, provided that $\Gamma(t)$ solves the $p$-GP hierarchy.

In order to simplify the presentation below, we introduce the notation

$$
\operatorname{Tr}^{1, m}:=\operatorname{Tr}_{1, k_{p}+1,2 k_{p}+1, \ldots,(m-1) k_{p}+1} .
$$

In the $L^{2}$ subcritical case, where $p<p_{L^{2}}=\frac{4}{d}$, we will now use the sequence of conserved quantities $\left(\left\langle\mathcal{K}^{(m)}\right\rangle_{\Gamma(t)}\right)_{m \in \mathbb{N}}$ to obtain an a priori bound on $\|\Gamma(t)\|_{\mathfrak{H}_{\xi}^{1}}$, for $\xi>0$ sufficiently small.

We define the Fourier multiplication operator $P_{j ; i}^{(r)}$ acting on $f:\left(\mathbb{R}^{d}\right)^{k_{p}} \rightarrow \mathbb{R}$ by

$$
\widehat{\left(P_{j ; i}^{(r)} f\right)}\left(\underline{\xi}_{r}\right):=p_{j}\left(\xi_{i}\right) \widehat{f}\left(\underline{\xi}_{r}\right),
$$

with symbols $p_{j}\left(\xi_{i}\right)$ that are smooth and supported in $\frac{2}{3} 2^{j}<\left|\xi_{i}\right|<3\left(2^{j}\right)$, for $j>1$ and in $\left|\xi_{1}\right| \leq 3$ when $j=0$. Also we define $P_{\leq j ; i}^{(r)}=\sum_{k \leq j} P_{k ; i}^{(r)}$ (and denote the corresponding symbol by $p_{\leq j ; i}$.

Moreover, we define the Fourier multiplication operator $P_{j ; i}^{(r), \leq}$ via

$$
\left(\widehat{P_{j ; i}^{(r), \leq}} f\right)\left(\underline{\xi}_{r}\right):=p_{j}\left(\xi_{i}\right)\left[\prod_{\substack{\ell=2 \\ l \neq i}}^{r} p_{\leq j}\left(\xi_{\ell}\right)\right] \widehat{f}\left(\underline{\xi}_{r}\right)
$$

and the Fourier multiplication operator $P_{j ; i i^{\prime}}^{(r)}$ acting on $\gamma^{(r)}$ by

$$
P_{j ; i i^{\prime}}^{(r)}=P_{j ; i}^{(r), \leq} P_{j ; i^{\prime}}^{(r), \leq}
$$


Then we have

$$
\begin{aligned}
\operatorname{Tr} & \left.S^{\left(k_{p}, \alpha\right)} \gamma^{\left(k_{p}\right)}\right) \\
& \leq \sum_{j=0}^{\infty} \sum_{i=1}^{k_{p}} \operatorname{Tr}\left(P_{j ; i i}^{\left(k_{p}\right)} S^{\left(k_{p}, \alpha\right)} \gamma^{\left(k_{p}\right)}\right) \\
& =\sum_{j=0}^{\infty} k_{p} \operatorname{Tr}\left(P_{j ; i i}^{\left(k_{p}\right)} S^{\left(k_{p}, \alpha\right)} \gamma^{\left(k_{p}\right)}\right) \\
& =\sum_{j=0}^{\infty} k_{p} \int d \underline{\xi}_{k_{p}} p_{j}\left(\xi_{1}\right)\left(\prod_{\ell=2}^{k_{p}} p_{\leq j}\left(\xi_{\ell}\right)\right)\left(\prod_{k=1}^{k_{p}}\left(1+\left|\xi_{k}\right|^{2}\right)^{\alpha}\right) \widehat{\gamma}\left(\underline{\xi}_{k_{p}} ; \underline{\xi}_{k_{p}}\right) \\
& \leq \sum_{j=0}^{\infty} k_{p}\left(1+2^{2 j}\right)^{\alpha k_{p}} \int d \xi_{1} p_{j}\left(\xi_{1}\right) \int d \xi_{2} \ldots d \xi_{k_{p}} \widehat{\gamma}\left(\underline{\xi}_{k_{p}} ; \underline{\xi}_{k_{p}}\right) \\
& =\sum_{j=0}^{\infty} k_{p}\left(1+2^{2 j}\right)^{\alpha k_{p}} \int d \xi_{1} p_{j}\left(\xi_{1}\right) \widehat{\gamma}\left(\xi_{1} ; \xi_{1}\right) \\
& =\sum_{j=0}^{\infty} k_{p}\left(1+2^{2 j}\right)^{\alpha k_{p}} \operatorname{Tr}\left(P_{j ; 1} \gamma^{(1)}\right)
\end{aligned}
$$

where to obtain (6.32) we used symmetry and to obtain (6.33) we used admissibility of $\gamma^{\left(k_{p}\right)}$.

Hence, we find

$$
\begin{aligned}
& 2 \operatorname{Tr}\left(K_{1} \gamma^{\left(k_{p}\right)}\right) \\
& \geq \quad 2 \operatorname{Tr}\left(K_{1}^{(1)} \gamma^{\left(k_{p}\right)}\right)-\frac{2|\mu| C_{0}(\alpha)}{p+2} \operatorname{Tr}\left(S^{\left(k_{p}, \alpha\right)} \gamma^{\left(k_{p}\right)}\right) \\
& \geq \operatorname{Tr}\left(S^{(1,1)} \gamma^{(1)}\right)-\frac{2|\mu| C_{0}(\alpha) k_{p}}{p+2} \sum_{j=0}^{\infty}\left(1+2^{2 j}\right)^{\alpha k_{p}} \operatorname{Tr}\left(P_{j ; 1}^{(1)} \gamma^{(1)}\right) \\
& \geq \operatorname{Tr}\left(S^{(1,1)} \gamma^{(1)}\right)-|\mu| C_{0}(\alpha) \sum_{j=0}^{\infty}\left(1+2^{2 j}\right)^{-\left(1-\alpha k_{p}\right)} \operatorname{Tr}\left(\left(1+2^{2 j}\right) P_{j ; 1}^{(1)} \gamma^{(1)}\right) \\
& \geq \operatorname{Tr}\left(S^{(1,1)} \gamma^{(1)}\right)-\left(\sup _{j \geq 0} \operatorname{Tr}\left(\left(1+2^{2 j}\right) P_{j ; 1}^{(1)} \gamma^{(1)}\right)\right)|\mu| C_{0}(\alpha) \sum_{j=0}^{\infty}\left(1+2^{2 j}\right)^{-\left(1-\alpha k_{p}\right)} \\
& \geq\left(1-|\mu| C_{0}(\alpha) \sum_{j=0}^{\infty} 2^{-2 j\left(1-\alpha k_{p}\right)}\right) \operatorname{Tr}\left(S^{(1,1)} \gamma^{(1)}\right) \\
& =D \operatorname{Tr}\left(S^{(1,1)} \gamma^{(1)}\right)
\end{aligned}
$$

with

$$
D:=D(\alpha, p, d,|\mu|)=\left(1-|\mu| \frac{C_{0}(\alpha)}{1-4^{-\left(1-\alpha k_{p}\right)}}\right) .
$$

Here to obtain (6.35) we used (6.20), to obtain (6.36) we used (6.34), to obtain (6.37) we used the inequality about Littlewood-Paley operators

$$
\sup _{j \geq 0} \operatorname{Tr}\left(\left(1+2^{2 j}\right) P_{j ; 1}^{(1)} \gamma^{(1)}\right) \leq \operatorname{Tr}\left(S^{(1,1)} \gamma^{(1)}\right)
$$


and to obtain (6.38) we used the fact that we consider the $L^{2}$ subcritical problem, hence $\alpha k_{p}<1$.

Accordingly, we conclude that if $|\mu|$ is such that $D(\alpha, p, d,|\mu|)>0$, i.e.

$$
|\mu|<\frac{1-4^{-\left(1-\alpha k_{p}\right)}}{C_{0}(\alpha)}
$$

then

$$
\begin{aligned}
\left\|\gamma^{(1)}\right\|_{\mathfrak{h}^{1}} & =\operatorname{Tr}\left(S^{(1,1)} \gamma^{(1)}\right) \\
& \leq \frac{2}{D} \operatorname{Tr}\left(K_{1} \gamma^{\left(k_{p}\right)}\right) .
\end{aligned}
$$

Next, we generalize this inequality to the higher order energy functionals.

To this end, we observe that by iterating (6.38) we obtain

$$
\begin{aligned}
& \left\langle\mathcal{K}^{(m)}\right\rangle_{\Gamma(t)}=\operatorname{Tr}^{1, m}\left(K_{1} K_{k_{p}+1} \cdots K_{(m-1) k_{p}+1} \gamma^{\left(m k_{p}\right)}\right) \\
& \quad \geq \frac{1}{D} \operatorname{Tr}^{1, m}\left(K_{1} K_{k_{p}+1} \cdots K_{(m-2) k_{p}+1} K_{(m-1) k_{p}+1}^{(1)} \gamma^{\left(m k_{p}\right)}\right) \\
& \quad \geq \cdots \cdots \\
& \quad \geq\left(\frac{1}{D}\right)^{m} \operatorname{Tr}^{1, m}\left(K_{1}^{(1)} K_{k_{p}+1}^{(1)} \cdots K_{(m-1) k_{p}+1}^{(1)} \gamma^{\left(m k_{p}\right)}\right) \\
& =\left(\frac{1}{2 D}\right)^{m} \operatorname{Tr}^{1, m}\left(S^{\left(m k_{p}, 1\right)} \gamma^{\left(m k_{p}\right)}\right) \\
& =\left(\frac{1}{2 D}\right)^{m}\left\|\gamma^{(m)}\right\|_{\mathfrak{h}^{1}},
\end{aligned}
$$

using the admissibility of $\gamma^{\left(m k_{p}\right)}$ in order to obtain the last line.

Consequently,

$$
\begin{aligned}
\|\Gamma(t)\|_{\mathfrak{H}_{\xi}^{1}} & \leq \sum_{m=1}^{\infty} \xi^{m}\left\|\gamma^{(m)}\right\|_{\mathfrak{h}^{1}} \\
& \leq \sum_{m=1}^{\infty}(2 D \xi)^{m}\left\langle\mathcal{K}^{(m)}\right\rangle_{\Gamma(t)} \\
& =\sum_{m=1}^{\infty}(2 D \xi)^{m}\left\langle\mathcal{K}^{(m)}\right\rangle_{\Gamma(0)} .
\end{aligned}
$$

This concludes the proof.

\section{Global Well-POSEDNESS OF SOLUTiOnS IN $\mathcal{H}_{\xi}^{1}$}

In this section, we will use the the higher order energy functionals in order to enhance local to global wellposedness for solutions in the spaces $\mathcal{H}_{\xi}^{1}$ constructed in [8], for initial data in $\mathfrak{H}_{\xi}^{1}$. The local well-posedness result proven in [8] has the following form. 
Theorem 7.1. Let

$$
\alpha \in \mathfrak{A}(d, p):=\left\{\begin{array}{cc}
\left(\frac{1}{2}, \infty\right) & \text { if } d=1 \\
\left(\frac{d}{2}-\frac{1}{2(p-1)}, \infty\right) & \text { if } d \geq 2 \text { and }(d, p) \neq(3,2) \\
{[1, \infty)} & \text { if }(d, p)=(3,2) .
\end{array}\right.
$$

Then, there exists a constant $0<\eta<1$ such that for $0<\xi \leq \eta \xi^{\prime} \leq 1$, there exists a constant $T_{0}\left(d, p, \xi, \xi^{\prime}\right)>0$ such that the following holds. Let $I:=[0, T]$ for $0<T<T_{0}\left(d, p, \xi, \xi^{\prime}\right)$. Then, there exists a unique solution $\Gamma \in L_{t \in I}^{\infty} \mathcal{H}_{\xi}^{\alpha}$ of the p-GP hierarchy, with

$$
\|\widehat{B} \Gamma\|_{L_{t \in I}^{1} \mathcal{H}_{\xi}^{\alpha}}<C\left(T, \xi, \xi^{\prime}, d, p\right)\left\|\Gamma_{0}\right\|_{\mathcal{H}_{\xi^{\prime}}^{\alpha}}
$$

in the space

$$
\mathcal{W}^{\alpha}(I, \xi)=\left\{\Gamma \in L_{t \in I}^{\infty} \mathcal{H}_{\xi}^{\alpha} \mid \widehat{B}^{+} \Gamma, \widehat{B}^{-} \Gamma \in L_{t \in I}^{2} \mathcal{H}_{\xi}^{\alpha}\right\}
$$

for the initial condition $\Gamma(0)=\Gamma_{0} \in \mathcal{H}_{\xi^{\prime}}^{\alpha}$.

We will next prove the following theorem.

Theorem 7.2. Assume that one of the two following cases is given:

- Energy subcritical, defocusing $p$-GP hierarchy with $p<\frac{4}{d-2}$ and $\mu=+1$. $\xi, \xi^{\prime}$ satisfy (3.5).

- $L^{2}$ subcritical, focusing $p$-GP hierarchy with $p<\frac{4}{d}$ and $\mu<0$ with $|\mu|<$ $\frac{1-4^{-\left(1-\alpha k_{p}\right)}}{C_{0}(\alpha)}$ where $C_{0}(\alpha)$ is characterized in (6.20). $\xi$, $\xi^{\prime}$ satisfy (3.9).

Then, there exists $T>0$ such that for $I_{j}:=[j T,(j+1) T]$, with $j \in \mathbb{Z}$, there exists a unique global solution $\Gamma \in \cup_{j \in \mathbb{Z}} \mathcal{W}^{1}\left(I_{j}, \xi\right)$ of the $p$-GP hierarchy with initial condition $\Gamma(0)=\Gamma_{0} \in \mathcal{H}_{\xi^{\prime}}^{1}$, satisfying

$$
\|\Gamma(t)\|_{\mathcal{H}_{\xi}^{1}} \leq C\left\|\Gamma_{0}\right\|_{\mathfrak{H}_{\xi^{\prime}}^{1}}
$$

for all $t \in \mathbb{R}$, if $\Gamma(t)$ is positive semidefinite for all $t \in I_{j}, j \in \mathbb{Z}$.

Proof. We need to prove that the higher order energy functionals are conserved for solutions in Theorem 7.1

For $m \in \mathbb{N}$ fixed, we consider $\gamma^{\left(m k_{p}\right)}$. Letting $\left(\phi_{j}\right)$ be an orthonormal basis of $L^{2}\left(\mathbb{R}^{d m k_{p}}\right)$, we define

$$
\gamma_{J}^{\left(m k_{p}\right)}(t):=\sum_{j \in J} \lambda_{j}(t) \phi_{j}\left(t, \underline{x}_{m k_{p}}\right) \overline{\phi_{j}\left(t, \underline{x}_{m k_{p}}^{\prime}\right)}
$$

for any finite subset $J \subset \mathbb{Z}$, with $0 \leq \lambda_{j}(t) \leq 1$.

Furthermore, we decompose

$$
\mathbb{R}^{j m d}=\bigcup_{r \in \mathbb{Z}^{j m d}} Q_{r}
$$

into disjoint cubes $Q_{r}$ obtained from translating the unit cube $[0,1)^{j m d}$ by $r \in \mathbb{Z}^{j m d}$. We then define

$$
\widetilde{\gamma}_{r, r^{\prime} ; J}^{(j)}:=P_{Q_{r}, Q_{r^{\prime}}}\left(\widetilde{\gamma}_{J}^{(j)}\right)
$$


where $P_{Q_{r}, Q_{r^{\prime}}}$ is the Fourier multiplication operator with symbol given by the characteristic function of $Q_{r} \times Q_{r^{\prime}}$.

Accordingly, letting $\ell=1$, we let

$$
\widetilde{\gamma}_{r, r_{p}^{\prime} ; J}^{\left(k_{p}\right)}\left(\underline{x}_{k_{p}} ; \underline{x}_{k_{p}}^{\prime}\right):=\operatorname{Tr}_{k_{p}+1, \ldots, m k_{p}}\left(K_{k_{p}+1} \cdots K_{(m-1) k_{p}+1} \gamma_{r, r^{\prime} ; J}^{\left(m k_{p}\right)}\right)
$$

and

$$
\begin{aligned}
& \widetilde{\gamma}_{r, r^{\prime} ; J}^{\left(2 k_{p}-1\right)}\left(\underline{x}_{k_{p}}, \underline{y}_{k_{p}-1} ; \underline{x}_{k_{p}}^{\prime}, \underline{y}_{k_{p}-1}^{\prime}\right) \\
& \left.\left.\quad:=\operatorname{Tr}_{k_{p}+1, \ldots, m k_{p}}\left(K_{k_{p}+1} \cdots K_{(m-1) k_{p}+1}\right)\right\} \gamma_{r, r^{\prime} ; J}^{\left(m k_{p}+\frac{p}{2}\right)}\right)\left(\underline{x}_{k_{p}}, \underline{y}_{k_{p}-1} ; \underline{x}_{k_{p}}^{\prime}, \underline{y}_{k_{p}-1}^{\prime}\right)
\end{aligned}
$$

where $y_{i}=x_{m k_{p}+i}$ and $y_{i}^{\prime}=x_{m k_{p}+i}^{\prime}$, for $i \in\left\{1, \ldots, k_{p}-1\right\}$.

Then, we define, similarly to the notation used in the proof of Theorem 4.1

$$
\begin{aligned}
& A_{h}^{(1)}\left(r, r^{\prime} ; J\right):=\operatorname{Tr}_{1}\left(K_{1}^{(1)} h_{1}^{ \pm} \widetilde{\gamma}_{r, r^{\prime} ; J}^{\left(k_{p}\right)}\right) \\
& A_{b}^{(1)}\left(r, r^{\prime} ; J\right):=\operatorname{Tr}_{1}\left(K_{1}^{(1)} b_{1}^{ \pm} \widetilde{\gamma}_{r, r^{\prime} ; J}^{\left(2 k_{p}-1\right)}\right) \\
& A_{h}^{(2)}\left(r, r^{\prime} ; J\right):=\operatorname{Tr}_{1}\left(K_{1}^{(2)} h_{1}^{ \pm} \operatorname{Tr}_{k_{p}+1 \cdots 2 k_{p}-1} \widetilde{\gamma}_{r, r^{\prime} ; J}^{\left(2 k_{p}-1\right)}\right) \\
& A_{b}^{(2)}\left(r, r^{\prime} ; J\right):=\operatorname{Tr}_{1}\left(K_{1}^{(2)} b_{1}^{ \pm} \widetilde{\gamma}_{r, r^{\prime} ; J}^{\left(2 k_{p}-1\right)}\right)
\end{aligned}
$$

we find that each of these terms can be written in the form

$$
A_{\sigma}^{(i)}\left(r, r^{\prime} ; J\right)=A_{\sigma,+}^{(i)}\left(r, r^{\prime} ; J\right)-A_{\sigma,-}^{(i)}\left(r, r^{\prime} ; J\right)
$$

for $\sigma \in\{h, b\}$ and $i \in\{1,2\}$. Following the arguments leading to the proof of Theorem 4.1, each term in the difference can be estimated by

$$
\begin{aligned}
& \left|A_{\sigma, \nu}^{(i)}\left(r, r^{\prime} ; J\right)\right| \\
& \quad<C\left(\operatorname{Tr}\left(\widetilde{\gamma}_{r, r ; J}^{(j)}\right)+\operatorname{Tr}\left(\widetilde{\gamma}_{r^{\prime}, r^{\prime} ; J}^{(j)}\right)\right) \\
& \quad<C|J|^{1 / 2}\left(\left[\operatorname{Tr}\left(\left|\widetilde{\gamma}_{r, r ; J}^{(j)}\right|^{2}\right)\right]^{1 / 2}+\operatorname{Tr}\left(\left[\widetilde{\gamma}_{r^{\prime}, r^{\prime} ; J}^{(j)}\right)\right]^{1 / 2}\right) \\
& \quad<2 C|J|^{1 / 2}\left\|\widetilde{\gamma}^{(j)}\right\|_{L^{2}\left(\mathbb{R}^{d j} \times \mathbb{R}^{d j}\right)} \\
& \quad<2 C|J|^{1 / 2}\left\|\gamma^{\left(m k_{p}\right)}\right\|_{H^{1}\left(\mathbb{R}^{d m k_{p}} \times \mathbb{R}^{d m k_{p}}\right)},
\end{aligned}
$$

where $\nu \in\{+,-\}$ and $j \in\left\{k_{p}, 2 k_{p}-1\right\}$. By the local wellposedness of the solution in $\mathcal{H}_{\xi}^{1}$, the last line is bounded. Therefore, all terms in (7.10) are well-defined, and similarly as in the proof of Theorem 4.1, they cancel,

$$
A_{h}^{(1)}\left(r, r^{\prime} ; J\right)+A_{h}^{(2)}\left(r, r^{\prime} ; J\right)+\mu A_{b}^{(1)}\left(r, r^{\prime} ; J\right)+A_{b}^{(2)}\left(r, r^{\prime} ; J\right)=0
$$

for all $r, r^{\prime} \in \mathbb{Z}^{d m k_{p}}$, and all $J$ with $|J|<\infty$. This implies that as in (4.11),

$$
\begin{aligned}
\partial_{t}\left\langle\mathcal{K}^{(m)}\right\rangle_{\Gamma(t)} & =\sum_{J \in \mathcal{I}} \sum_{r, r^{\prime}}\left(A_{h}^{(1)}\left(r, r^{\prime} ; J\right)+A_{h}^{(2)}\left(r, r^{\prime} ; J\right)+\mu A_{b}^{(1)}\left(r, r^{\prime} ; J\right)+A_{b}^{(2)}\left(r, r^{\prime} ; J\right)\right) \\
& =0
\end{aligned}
$$

where $\mathbb{Z}=\cup_{J \in \mathcal{I}} J$ is an arbitrary decomposition of $\mathbb{Z}$ into mutually disjoint discrete intervals $J$ of finite size. 
Accordingly, if the solution $\Gamma(t) \in \mathcal{H}_{\xi}^{1}$ obtained from Theorem 7.1 has initial data in the subspace $\Gamma(0)=\Gamma_{0} \in \mathfrak{H}_{\xi^{\prime}}^{1} \subset \mathcal{H}_{\xi^{\prime}}^{1}$, then

$$
\begin{aligned}
\left\langle\mathcal{K}^{(m)}\right\rangle_{\Gamma(t)} & =\left\langle\mathcal{K}^{(m)}\right\rangle_{\Gamma_{0}}+\int_{0}^{t} d s \partial_{s}\left\langle\mathcal{K}^{(m)}\right\rangle_{\Gamma(s)} \\
& =\left\langle\mathcal{K}^{(m)}\right\rangle_{\Gamma_{0}},
\end{aligned}
$$

for all $m \in \mathbb{N}$. That is, the higher order energy functionals are conserved.

In the case $p<\frac{4}{d-2}$ (energy subcritical), and $\mu=+1$ (defocusing), we choose $\xi_{1}, \xi$ such that

$$
0<\xi_{1} \leq \eta \xi \leq \eta\left(1+\frac{2}{p+2} C_{S o b}(d, p)\right)^{-\frac{1}{k_{p}}} \xi^{\prime} .
$$

where $0<\eta<1$ is the constant in Theorem 7.1. Next, we pick

$$
T:=\min \left\{T\left(d, p, \xi_{1}, \xi\right), T\left(d, p, \xi, \xi^{\prime}\right)\right\},
$$

where $T\left(d, p, \xi, \xi^{\prime}\right)$ is defined as in Theorem 7.1 .

Then, Theorem 6.2 implies that the a priori bound

$$
\begin{aligned}
\|\Gamma(t)\|_{\mathcal{H}_{\xi}^{1}} & \leq\|\Gamma(t)\|_{\mathfrak{H}_{\xi}^{1}} \leq \sum_{m \in \mathbb{N}}(2 \xi)^{m}\left\langle\mathcal{K}^{(m)}\right\rangle_{\Gamma(t)} \\
& =\sum_{m \in \mathbb{N}}(2 \xi)^{m}\left\langle\mathcal{K}^{(m)}\right\rangle_{\Gamma_{0}} \leq\|\Gamma(0)\|_{\mathfrak{H}_{\xi^{\prime}}^{1}}
\end{aligned}
$$

holds for $t \in I_{0}=[0, T]$.

Next, Theorem 7.1 implies that there exists a unique solution in $\mathcal{W}^{1}\left(I_{1}, \xi_{1}\right)$ for $t \in I_{1}=[T, 2 T]$, for the initial condition $\Gamma(T) \in \mathcal{H}_{\xi}^{1}$. Therefore, we have for every $m \in \mathbb{N}$,

$$
\left\langle\mathcal{K}^{(m)}\right\rangle_{\Gamma(t)}=\left\langle\mathcal{K}^{(m)}\right\rangle_{\Gamma(T)}=\left\langle\mathcal{K}^{(m)}\right\rangle_{\Gamma_{0}}
$$

for all $t \in I_{1}$. Since these are $t$-independent $\mathbb{C}$-numbers, this implies that

$$
\begin{aligned}
\|\Gamma(t)\|_{\mathcal{H}_{\xi}^{1}} & \leq\|\Gamma(t)\|_{\mathfrak{H}_{\xi}^{1}} \leq \sum_{m \in \mathbb{N}}(2 \xi)^{m}\left\langle\mathcal{K}^{(m)}\right\rangle_{\Gamma(t)} \\
& =\sum_{m \in \mathbb{N}}(2 \xi)^{m}\left\langle\mathcal{K}^{(m)}\right\rangle_{\Gamma_{0}} \leq\|\Gamma(0)\|_{\mathfrak{H}_{\xi^{\prime}}^{1}}
\end{aligned}
$$

for $t \in I_{1}$. In particular, this is true for the same value of $\xi$ as above, for the case of the interval $I_{0}$. Thus, by bootstrapping, we find that instead of $\Gamma(t) \in \mathcal{H}_{\xi_{1}}^{1}$, one in fact has $\Gamma(t) \in \mathcal{H}_{\xi}^{1}$ for all $t \in[T, 2 T]$, where $\xi_{1} \leq \eta \xi$.

Again invoking Theorem 7.1 and repeating the above, we furthermore conclude that there exists a unique solution $\Gamma \in \mathcal{W}^{1}\left(I_{1}, \xi_{1}\right)$ for $t \in I_{2}=[2 T, 3 T]$ for the initial condition $\Gamma(2 T) \in \mathcal{H}_{\xi}^{1}$. Accordingly, higher order energy conservation shows that $\Gamma(t) \in \mathcal{H}_{\xi}^{1}$ for all $t \in I_{2}$.

Iterating this argument, we have proved that there exists a unique global solution $\Gamma \in \cup_{j \in \mathbb{Z}} \mathcal{W}^{1}\left(I_{j}, \xi_{1}\right)$ of the $p$-GP hierarchy with initial condition $\Gamma(0)=\Gamma_{0} \in \mathcal{H}_{\xi^{\prime}}^{1}$, satisfying

$$
\|\Gamma(t)\|_{\mathcal{H}_{\xi_{1}}^{1}}<\|\Gamma(t)\|_{\mathcal{H}_{\xi}^{1}} \leq\left\|\Gamma_{0}\right\|_{\mathfrak{H}_{\xi^{\prime}}^{1}}
$$


for all $t \in \mathbb{R}$.

In the case $p<\frac{4}{d}$ ( $L^{2}$ subcritical), and $\mu<0$ (focusing) with

$$
|\mu|<\frac{1-4^{-\left(1-\alpha k_{p}\right)}}{C_{0}(\alpha)}
$$

we use that

$$
\begin{aligned}
\|\Gamma(t)\|_{\mathcal{H}_{\xi}^{1}} & \leq\|\Gamma(t)\|_{\mathfrak{H}_{\xi}^{1}} \leq \sum_{m \in \mathbb{N}}(2 D \xi)^{m}\left\langle\mathcal{K}^{(m)}\right\rangle_{\Gamma(t)} \\
& =\sum_{m \in \mathbb{N}}(2 D \xi)^{m}\left\langle\mathcal{K}^{(m)}\right\rangle_{\Gamma(0)} \leq\|\Gamma(0)\|_{\mathfrak{H}_{\xi^{\prime}}^{1}}
\end{aligned}
$$

for all $t \in[0, T]$, and for $\xi, \xi^{\prime}$ satisfying (3.9). Accordingly, we can repeat the arguments for the case $p<\frac{4}{d-2}$ and $\mu=+1$. This completes the proof.

Acknowledgements. We are grateful to I. Rodnianski and M. Visan for very useful comments. We thank W. Beckner, B. Erdogan and N. Tzirakis for helpful discussions. The work of T.C. was supported by NSF grant DMS 0704031 / DMS-0940145, DMS-1009448, and DMS-1151414 (CAREER). The work of N.P. was supported NSF grant number DMS 0758247, DMS 1101192 and an Alfred P. Sloan Research Fellowship.

\section{REFERENCES}

[1] R. Adami, G. Golse, A. Teta, Rigorous derivation of the cubic NLS in dimension one, J. Stat. Phys. 127, no. 6, 1194-1220 (2007).

[2] M. Aizenman, E.H. Lieb, R. Seiringer, J.P. Solovej, J. Yngvason Bose-Einstein Quantum Phase Transition in an Optical Lattice Model, Phys. Rev. A 70, 023612 (2004).

[3] I. Anapolitanos, I.M. Sigal, The Hartree-von Neumann limit of many body dynamics, Preprint http://arxiv.org/abs/0904.4514

[4] V. Bach, T. Chen, J. Fröhlich and I. M. Sigal, Smooth Feshbach map and operator-theoretic renormalization group methods, J. Funct. Anal., 203 (1), 44-92 (2003).

[5] W. Beckner, Multilinear embedding estimates for the fractional Laplacian, Preprint http://arxiv.org/abs/1004.2259

[6] T. Cazenave, Semilinear Schrödinger equations, Courant lecture notes 10, Amer. Math. Soc. (2003).

[7] T. Chen, N. Pavlović, The quintic NLS as the mean field limit of a Boson gas with three-body interactions, J. Funct. Anal., 260 (4), 959-997, 2011.

[8] T. Chen, N. Pavlović, On the Cauchy problem for focusing and defocusing Gross-Pitaevskii hierarchies, Discr. Contin. Dyn. Syst., 27 (2), 715 - 739, 2010.

[9] T. Chen, N. Pavlović, N. Tzirakis, Energy conservation and blowup of solutions for focusing GP hierarchies, Ann. Inst. H. Poincare (C) Anal. Non-Lin., 27 (5), 1271-1290, 2010.

[10] A. Elgart, L. Erdös, B. Schlein, H.-T. Yau, Gross-Pitaevskii equation as the mean field limit of weakly coupled bosons, Arch. Rat. Mech. Anal. 179, no. 2, 265-283 (2006).

[11] L. Erdös, B. Schlein, H.-T. Yau, Derivation of the Gross-Pitaevskii hierarchy for the dynamics of Bose-Einstein condensate, Comm. Pure Appl. Math. 59 (12), 1659-1741 (2006).

[12] L. Erdös, B. Schlein, H.-T. Yau, Derivation of the cubic non-linear Schrödinger equation from quantum dynamics of many-body systems, Invent. Math. 167 (2007), 515-614.

[13] L. Erdös, H.-T. Yau, Derivation of the nonlinear Schrödinger equation from a many body Coulomb system, Adv. Theor. Math. Phys. 5, no. 6, 1169-1205 (2001).

[14] J. Fröhlich, S. Graffi, S. Schwarz, Mean-field- and classical limit of many-body Schrödinger dynamics for bosons, Comm. Math. Phys. 271, no. 3, 681-697 (2007).

[15] J. Fröhlich, A. Knowles, A. Pizzo, Atomism and quantization, J. Phys. A 40, no. 12, 30333045 (2007). 
[16] J. Fröhlich, A. Knowles, S. Schwarz On the Mean-Field Limit of Bosons with Coulomb TwoBody Interaction, Comm. Math. Phys. 288 (3), 10231059 (2009).

[17] M. Grillakis, M. Machedon, A. Margetis, Second-order corrections to mean field evolution for weakly interacting Bosons. I, Comm. Math. Phys. 294 (1), 273301 (2010).

[18] M. Grillakis, A. Margetis, A priori estimates for many-body Hamiltonian evolution of interacting boson system, J. Hyperbolic Differ. Equ. 5 (4), 857-883 (2008).

[19] K. Hepp, The classical limit for quantum mechanical correlation functions, Comm. Math. Phys. 35, 265-277 (1974).

[20] S. Klainerman, M. Machedon, On the uniqueness of solutions to the Gross-Pitaevskii hierarchy, Commun. Math. Phys. 279, no. 1, 169-185 (2008).

[21] K. Kirkpatrick, B. Schlein, G. Staffilani, Derivation of the two dimensional nonlinear Schrödinger equation from many body quantum dynamics, Amer. J. Math. 133 (1), 91130 (2011).

[22] E.H. Lieb, R. Seiringer, Proof of Bose-Einstein condensation for dilute trapped gases, Phys. Rev. Lett. 88, 170409 (2002).

[23] E.H. Lieb, R. Seiringer, J.P. Solovej, J. Yngvason, The mathematics of the Bose gas and its condensation, Birkhäuser (2005).

[24] E.H. Lieb, R. Seiringer, J. Yngvason, A rigorous derivation of the Gross-Pitaevskii energy functional for a two-dimensional Bose gas, Commun. Math. Phys. 224 (2001).

[25] I. Rodnianski, B. Schlein, Quantum fluctuations and rate of convergence towards mean field dynamics, preprint arXiv:math-ph/0711.3087.

[26] B. Schlein, Derivation of Effective Evolution Equations from Microscopic Quantum Dynam$i c s$, Lecture notes for the minicourse held at the 2008 CMI Summer School in Zurich.

[27] H. Spohn, Kinetic Equations from Hamiltonian Dynamics, Rev. Mod. Phys. 52, no. 3, 569615 (1980).

[28] T. Tao, Nonlinear dispersive equations. Local and global analysis, CBMS 106, eds: AMS, 2006.

T. Chen, Department of Mathematics, University of Texas at Austin.

E-mail address: tc@math.utexas.edu

N. Pavlović, Department of Mathematics, University of Texas at Austin.

E-mail address: natasa@math.utexas.edu 